\title{
VIABILITY AND ARBITRAGE UNDER KNIGHTIAN UNCERTAINTY
}

\author{
MATTEO BURZONI \\ Department of Mathematics, Università degli studi di Milano \\ FRANK RIEDEL \\ Center for Mathematical Economics, Bielefeld University and School of Economics, College of Business and \\ Economics, University of Johannesburg
}

H. METE SONER

Department of Operations Research and Financial Engineering, Princeton University

\begin{abstract}
We reconsider the microeconomic foundations of financial economics. Motivated by the importance of Knightian uncertainty in markets, we present a model that does not carry any probabilistic structure ex ante, yet is based on a common order. We derive the fundamental equivalence of economic viability of asset prices and absence of arbitrage. We also obtain a modified version of the fundamental theorem of asset pricing using the notion of sublinear pricing measures. Different versions of the efficient market hypothesis are related to the assumptions one is willing to impose on the common order.
\end{abstract}

KEYWORDS: Viability, Knightian uncertainty, no arbitrage, robust finance.

\section{INTRODUCTION}

ASSET PRICING MODELS TYPICALLY TAKE a basic set of securities as given and determine the range of option prices that is consistent with the absence of arbitrage. From an economic point of view, it is crucial to know if modeling security prices directly is justified; an asset pricing model is called viable if its security prices can be thought of as (endogenous) equilibrium outcomes of a competitive economy. Traditional finance models rely on a probabilistic framework. The capital asset pricing model assumes that agents have meanvariance preferences and share the same view of mean and variance-covariance matrix of asset returns. The consumption-based capital asset pricing model derives asset returns from economic equilibrium and also assumes that agents share the same prior. Harrison and Kreps (1979) realized that a reference probability that determines the null sets, the topology, and the order of the model is sufficient to prove the equivalence of economic viability and pricing by arbitrage. The common prior or the weaker reference probability assumption is made in most asset pricing models.

Recently, a large and increasing body of literature has focused on decisions, markets, and economic interactions under uncertainty. Frank Knight's pioneering work (Knight (1921)) distinguishes risk-a situation that allows for an objective probabilistic

Matteo Burzoni: matteo.burzoni@unimi.it

Frank Riedel: frank.riedel@uni-bielefeld.de

H. Mete Soner: soner@princeton.edu

Comments by Rose-Anne Dana, Filipe Martins-da-Rocha, and seminar and workshop participants at Columbia University, Oxford University, the Institut Henri Poincaré Paris, and Padova University, are gratefully acknowledged. Matteo Burzoni acknowledges the support of the Hooke Research Fellowship. Matteo Burzoni and Mete Soner acknowledge the support of the ETH Foundation, the Swiss Finance Institute, and the Swiss National Foundation through SNF 200020_172815. Frank Riedel gratefully acknowledges the financial support of the German Research Foundation (DFG) via CRC 1283.

(C) 2021 The Authors. Econometrica published by John Wiley \& Sons Ltd on behalf of The Econometric Society. Frank Riedel is the corresponding author on this paper. This is an open access article under the terms of the Creative Commons Attribution-NonCommercial-NoDerivs License, which permits use and distribution in any medium, provided the original work is properly cited, the use is non-commercial and no modifications or adaptations are made. 
description-from uncertainty - a situation that cannot be modeled by a single probability distribution. By now, it is widely acknowledged that drift and volatility of asset prices, the term structure of interest rates, and credit risk are important instances in which the probability distribution of the relevant parameters is imprecisely known, if not completely unknown. Epstein and Ji (2013) emphasized the relevance of nonprobabilistic uncertainty in financial modeling when parameters vary too frequently to be estimated accurately, or when nonlinearities arise that are too complex to be captured by existing models, or when nonstationarities prevent the use of the law of large numbers or the central limit theorem. They show that a probability space framework is not able to model ambiguity about volatility. ${ }^{1}$

We take these insights as a motivation to reconsider the foundations of arbitrage pricing and its relation to economic equilibrium without imposing a priori a probability space framework. We show that the basic relations between economic equilibrium (viability), absence of arbitrage, and suitable pricing functionals can be proved with ease by merely assuming a common order with respect to which preferences are monotone. ${ }^{2}$ Using this approach, we achieve a unifying theory that covers classical models of risk as well as new models of ambiguity.

We first show that the absence of arbitrage and the (properly defined) economic viability of the model are equivalent. In equilibrium, there are no arbitrage opportunities; conversely, for arbitrage-free asset pricing models, it is possible to construct a heterogeneous agent economy such that the asset prices are equilibrium prices of that economy.

The second key result is the fundamental theorem of asset pricing. In contrast to risk, it is no longer possible to characterize viability through the existence of a single linear pricing measure (or equivalent martingale measure). Instead, it is necessary to use a suitable nonlinear pricing expectation, that we call a sublinear martingale expectation. A sublinear expectation has the common properties of an expectation including monotonicity, preservation of constants, and positive homogeneity, yet it is no longer additive. Indeed, sublinear expectations can be represented as the supremum of a class of (linear) expectations, an operation that does not preserve linearity. ${ }^{3}$ Nonlinear expectations arise in decision-theoretic models of ambiguity-averse preferences (Gilboa and Schmeidler (1989), Maccheroni, Marinacci, and Rustichini (2006)). It is interesting to see that a similar nonlinearity arises here for the pricing functional. A general theory of equilibrium with such sublinear prices is developed in Beissner and Riedel (2019). ${ }^{4}$

The common order shapes equilibrium asset prices. We study various common orders and how they are related to versions of the efficient market hypothesis (Fama (1970)). A strong interpretation of the efficient market hypothesis says that properly discounted expected returns of assets are equal to the return of a safe bond. We obtain this conclusion when the common order is based on expected payoffs with respect to a common

\footnotetext{
${ }^{1}$ Compare also the general conceptual discussion of ambiguity and limitations of probabilistic modeling in Lo and Mueller (2010).

${ }^{2}$ An obvious and intuitive example of an order that we have in mind here is the pointwise order, that is, when agents will prefer a contingent consumption plan over their endowment if the new plan pays off more in every state of the world. Preferences over monetary outcomes are naturally assumed to be monotone with respect to this basic order.

${ }^{3}$ In economics, such a representation theorem appears first in Gilboa and Schmeidler (1989). Sublinear expectations also arise in robust statistics (compare Huber (1981)), and they play a fundamental role in theory of risk measures in Finance; see Artzner, Delbaen, Eber, and Heath (1999) and Föllmer and Schied (2011).

${ }^{4}$ Given that we have a nonlinear price system, one might wonder whether agents can generate arbitrage gains by splitting a consumption bundle into two or more plans. The convexity of our price functional excludes such arbitrage opportunities; see Proposition 1 in Beissner and Riedel (2019).
} 
prior. When the common order is given by the almost sure order under a common prior, one obtains a weaker version of the efficient market hypothesis: under an equivalent pricing measure, expected returns are equal..$^{5}$ In situations of Knightian uncertainty, different specifications of the common order can be made. An example is the quasi-sure order induced by a set of priors: a claim dominates quasi-surely another claim if it is almost surely greater or equal under all considered probability measures. Another example is the order induced by smooth ambiguity preferences, as introduced by Klibanoff, Marinacci, and Mukerji (2005), where Knightian uncertainty is modeled by a second-order prior over a class of multiple priors. We show that weaker versions of the efficient market hypothesis hold, depending on the strength of the assumptions we are willing to impose on the common order, and how the related fundamental theorem of asset pricing needs to be suitably adapted.

\section{Further Related Literature}

The relation of arbitrage and viability has been discussed in various contexts. Jouini and Kallal (1995) and Jouini and Kallal (1999) discussed models with transaction cost and other frictions. Werner (1987) and Dana, Le Van, and Magnien (1999) studied the absence of arbitrage in its relation to equilibrium when a finite set of agents is fixed a priori whereas Cassese (2017) characterized the absence of arbitrage in an order-theoretic framework derived from coherent risk measures. Knightian uncertainty is also closely related to robustness concerns that play an important role in macroeconomic models that deal with the fear of model misspecification (Hansen and Sargent (2001, 2008)). The pointwise order corresponds to the "model-independent" (or rather "probability-free") approach in finance that has been discussed, for example, in Riedel (2015), Acciaio, Beiglböck, Penkner, and Schachermayer (2016), Burzoni, Frittelli, Hou, Maggis, and Obłój (2019), and Bartl, Cheridito, Kupper, and Tangpi (2017). This literature uses different notions of "relevant payoffs" that our approach allows to unify under a common framework.

The paper is set up as follows. Section 2 describes the model, the two main contributions of this paper, and provides four examples. Section 3 derives various classic and new forms of the efficient market hypothesis. The modeling philosophy is discussed in more detail in Section 4. Section 5 is devoted to the proofs of the main theorems. The supplementary online material contains a detailed study of general discrete time markets when the space of contingent payoffs consists of bounded measurable functions. It also discusses further extensions as, for example, the equivalence of absence of arbitrage and absence of free lunches with vanishing risk, or the question if an optimal superhedge for a given claim exists.

\section{A PROBABILITY-FREE FOUNDATION FOR FINANCIAL ECONOMICS}

A nonempty set $\Omega$ contains the states of the world; the $\sigma$-field $\mathcal{F}$ on $\Omega$ collects the possible events.

The commodity space (of contingent claims) $\mathcal{H}$ is a vector space of $\mathcal{F}$-measurable realvalued functions containing all constant functions. We will use the symbol $c$ both for real numbers as well as for constant functions. $\mathcal{H}$ is endowed with a metrizable topology $\tau$ and a preorder $\leq$ that are compatible with the vector space operations.

\footnotetext{
${ }^{5}$ This statement is equivalent to the classic version of the fundamental theorem of asset pricing in Harrison and Kreps (1979), Harrison and Pliska (1981), Duffie and Huang (1985), Dalang, Morton, and Willinger (1990), Delbaen and Schachermayer (1998).
} 
The abstract vector space model allows to cover the typical models that have been used in financial economics. Under risk, it is common to take a space of suitably integrable functions with respect to a given prior with the usual almost sure order; without an ex ante given probability measure, integrability cannot be used as a criterion. We thus allow for more generality here in order to include, for example, spaces of suitably bounded measurable functions.

We assume throughout that agents' preferences are monotone with respect to the preorder $\leq$ which thus plays a crucial role in our analysis. A major conclusion of our study is that the strength of the assumptions we are willing to make on the common order (and, therefore, on the agents populating the economy) shapes the results about market returns as we shall see in detail in Section 3. We assume throughout that the preorder $\leq$ is consistent with the order on the reals for constant functions and with the pointwise order for measurable functions. A consumption plan $Z \in \mathcal{H}$ is negligible if we have $0 \leq Z$ and $Z \leq 0 . C \in \mathcal{H}$ is nonnegative if $0 \leq C$ and positive if in addition not $C \leq 0$. We denote by $\mathcal{Z}, \mathcal{P}$ and $\mathcal{P}^{+}$the class of negligible, nonnegative, and positive contingent claims, respectively.

We introduce a class of relevant contingent claims $\mathcal{R}$, a convex subset of $\mathcal{P}^{+}$. The relevant claims are used below in two important ways. On the one hand, they describe the nonnegative consumption plans that some agents strictly prefer to the null claim. On the other hand, they signal arbitrage: if a net trade allows to obtain a payoff that dominates a relevant payoff with respect to the common order, we speak of an arbitrage. In the spirit of Arrow (1953) and most of the literature, a common choice of the relevant claims is the set of positive claims $\mathcal{P}^{+}$; we invite the reader to make this identification at first reading. However, it might be of interest to consider smaller relevant sets in some economic contexts. The introduction of $\mathcal{R}$ also allows to subsume various notions of arbitrage that appeared in the literature, compare the discussion in Section 4.

The financial market is modeled by the set of net trades $\mathcal{I} \subset \mathcal{H}$, a convex cone containing $0 . \mathcal{I}$ is the set of payoffs that the agents can achieve from zero initial wealth by trading in the financial market. In the basic frictionless model of securities, $\mathcal{I}$ contains the payoffs of self-financing strategies with zero initial capital. In a frictionless market, $\mathcal{I}$ is a subspace. When short selling constraints, credit line limitations, or transaction costs are imposed, for example, we are led to a convex cone instead of a subspace; see Example 4.1.

An agent in this economy is described by a preference relation $\preceq$ (i.e., a complete and transitive binary relation) on $\mathcal{H}$, that is,

- weakly monotone with respect to $\leq$, that is, $X \leq Y$ implies $X \preceq Y$ for every $X, Y \in \mathcal{H}$;

- convex, that is, the upper contour sets $\{Z \in \mathcal{H}: Z \succeq X\}$ are convex;

- $\tau$-lower semicontinuous, that is, for every sequence $\left\{X_{n}\right\}_{n=1}^{\infty} \subset \mathcal{H}$ converging to $X$ in $\tau$ with $X_{n} \preceq Y$ for $n \in \mathbb{N}$, we have $X \preceq Y$.

The set of all agents is denoted by $\mathcal{A}$.

In the spirit of Harrison and Kreps (1979), we think of a potentially large set of agents about whom some things are known, without assuming that we know exactly their preferences or their number. We only impose a list of properties on preferences that are standard in economics. In particular, bearing in mind the interpretation of $\leq$ as a common order, the preferences are monotone with respect to $\leq$. Moreover, we impose some weak form of continuity with respect to the given topology $\tau$; it is known that, in general, some form of continuity is required for the existence of equilibrium. Convexity reflects a preference for diversification. We stress that preferences are defined on the entire commodity space $\mathcal{H}$; this assumption can be relaxed (we refer to the Supplementary Online Material (Burzoni, Riedel, and Soner (2021)) for the technical details). 
A financial market $(\mathcal{H}, \tau, \leq, \mathcal{I}, \mathcal{R})$ is viable if there is a family of agents $\left\{\preceq_{a}\right\}_{a \in A} \subset \mathcal{A}$ such that

- 0 is optimal for each agent $a \in A$, that is,

$$
\forall \ell \in \mathcal{I} \quad \ell \preceq_{a} 0,
$$

- for every relevant claim $R \in \mathcal{R}$ there exists an agent $a \in A$ such that

$$
0 \prec_{a} R \text {. }
$$

We say that $\left\{\preceq_{a}\right\}_{a \in A}$ supports the financial market $(\mathcal{H}, \tau, \leq, \mathcal{I}, \mathcal{R})$.

A market is in equilibrium when agents have no incentive to trade away from their current endowment. ${ }^{6}$ We generalize the viability definition of Harrison and Kreps (1979) who use a single representative agent to allow for economies with heterogeneous agents. Heterogeneity of agents allows us to prove the two main theorems in great generality with easy arguments without the need to construct strictly monotone preferences (that might not exist in all cases). We replace the strict monotonicity assumption by the weaker Condition (2.2) which, in particular, excludes the trivial case of agents who are indifferent between all payoffs. Compare also our discussion of the viability concept in Section 4 below.

A net trade $\ell \in \mathcal{I}$ is an arbitrage if there exists a relevant claim $R^{*} \in \mathcal{R}$ such that $\ell \geq$ $R^{*}$. More generally, a sequence of net trades $\left\{\ell_{n}\right\}_{n=1}^{\infty} \subset \mathcal{I}$ is a free lunch with vanishing risk if there exists a relevant claim $R^{*} \in \mathcal{R}$ and a sequence $\left\{e_{n}\right\}_{n=1}^{\infty} \subset \mathcal{H}$ of nonnegative consumption plans with $e_{n} \stackrel{\tau}{\rightarrow} 0$ satisfying $e_{n}+\ell_{n} \geq R^{*}$ for all $n \in \mathbb{N}$. We say that the financial market is strongly free of arbitrage if there is no free lunch with vanishing risk. In general, the absence of arbitrage is not equivalent to the absence of free lunches with vanishing risk. ${ }^{7}$ In the Supplementary Online Material, we establish the equivalence for finite horizon discrete time financial markets.

\section{Two Theorems}

Our first theorem establishes the equivalence of viability and absence of arbitrage.

THEOREM 2.1: A financial market is strongly free of arbitrage if and only if it is viable.

In the standard literature, the model of the economy is constructed on a probability space with a given reference probability $\mathbb{P}$ that determines the null sets and the topology of the model. In such common prior models, a financial market is viable if and only if there exists a linear pricing measure in the form of a risk-neutral probability measure $\mathbb{P}^{*}$ that is equivalent to $\mathbb{P}$, as Harrison and Kreps (1979) have shown. In the absence of a common prior, we have to work with a more general, sublinear notion of pricing. A functional

$$
\mathcal{E}: \mathcal{H} \rightarrow \mathbb{R} \cup\{\infty\}
$$

\footnotetext{
${ }^{6}$ We take the endowment to be zero in our definition; this comes without loss of generality; see also the discussion in Section 4.

${ }^{7}$ Our notion of free lunch with vanishing risk is a modification of the one used by Kreps (1981). It corresponds to the notion proposed by Delbaen and Schachermayer (1998) for financial markets in continuous time in a probability space framework. This notion and our adapted viability concept allow to prove the equivalence of viability, absence of arbitrage, and the existence of a suitable pricing functional in contrast to Kreps (1981).
} 
is a sublinear expectation if it is monotone with respect to $\leq$, translation-invariant, that is, $\mathcal{E}(X+c)=\mathcal{E}(X)+c$ for all constant claims $c \in \mathcal{H}$ and $X \in \mathcal{H}$, and sublinear, that is, for all $X, Y \in \mathcal{H}$ and $\lambda>0$, we have $\mathcal{E}(X+Y) \leq \mathcal{E}(X)+\mathcal{E}(Y)$ and $\mathcal{E}(\lambda X)=\lambda \mathcal{E}(X)$. $\mathcal{E}$ has full support if $\mathcal{E}(R)>0$ for every $R \in \mathcal{R}$. Last but not least, $\mathcal{E}$ has the martingale property if $\mathcal{E}(\ell) \leq 0$ for every $\ell \in \mathcal{I}$. We say in short that $\mathcal{E}$ is a sublinear martingale expectation with full support if all the previous properties are satisfied.

It is well known from decision theory that sublinear expectations can be written as upper expectations over a set of probability measures. In our more abstract framework, probability measures are replaced by suitably normalized functionals. We say that $\varphi \in \mathcal{H}_{+}^{\prime}{ }^{8}$ is a martingale functional ${ }^{9}$ if it satisfies $\varphi(1)=1$ (normalization) and $\varphi(\ell) \leq 0$ for all $\ell \in \mathcal{I}$. In the spirit of the probabilistic language, we call a linear functional absolutely continuous if it assigns the value zero to all negligible claims. We denote by $\mathcal{Q}_{\text {ac }}$ the set of absolutely continuous martingale functionals.

The notions that we introduced now allow us to state the general version of the fundamental theorem of asset pricing in our order-theoretic context.

THEOREM 2.2-Fundamental theorem of asset pricing: The financial market is viable if and only if there exists a lower semicontinuous sublinear martingale expectation with full support.

In a viable market, the set of absolutely continuous martingale functionals $\mathcal{Q}_{\mathrm{ac}}$ is not empty and

$$
\mathcal{E}_{\mathcal{Q}_{\mathrm{ac}}}(X):=\sup _{\phi \in \mathcal{Q}_{\mathrm{ac}}} \phi(X)
$$

is a lower semicontinuous sublinear martingale expectation with full support. Moreover, $\mathcal{E}_{\mathcal{Q}_{\mathrm{ac}}}$ is maximal, in the sense that any other lower semicontinuous sublinear martingale expectation with full support $\mathcal{E}$ satisfies $\mathcal{E}(X) \leq \mathcal{E}_{\mathcal{Q}_{\mathrm{ac}}}(X)$ for all $X \in \mathcal{H}$.

REMARK 2.3: Under nonlinear expectations, one has to distinguish martingales from symmetric martingales; a symmetric martingale has the property that the process itself and its negative are martingales. In our context, this condition translates to the equality $\mathcal{E}_{\mathcal{Q}_{\mathrm{ac}}}(\ell)=0$ for all $\ell \in \mathcal{I}$. When the set of net trades $\mathcal{I}$ is a linear space as in the case of frictionless markets, a net trade $\ell$ and its negative $-\ell$ belong to $\mathcal{I}$. In this case, sublinearity and the condition $\mathcal{E}_{\mathcal{Q}_{\mathrm{ac}}}(\ell) \leq 0$ for all $\ell \in \mathcal{I}$ imply $\mathcal{E}_{\mathcal{Q}_{\mathrm{ac}}}(\ell)=0$ for all net trades $\ell \in \mathcal{I}$. Thus, the net trades $\ell$ are symmetric martingales.

\section{Four Examples}

Our novel approach builds the foundations of financial economics without imposing any a priori probabilistic structure, thereby including the new paradigm of Knightian uncertainty into the theory. We illustrate the unifying power of the model with four examples ranging from classical situations of risk to new ones with ambiguity.

EXAMPLE 2.4-The atom of finance and complete markets: The basic one-step binomial model, that we like to call the atom of finance, and consists of two states of the world,

\footnotetext{
${ }^{8} \mathcal{H}^{\prime}$ is the topological dual of $\mathcal{H}$ and $\mathcal{H}_{+}^{\prime}$ is the set of positive elements in $\mathcal{H}^{\prime}$.

${ }^{9}$ In this generality the terminology, functional is more appropriate. When the dual space $\mathcal{H}^{\prime}$ can be identified with a space of measures, we will use the terminology martingale measure. The technical question whether these measures are countably additive is discussed in the Supplementary Online Material.
} 
$\Omega=\{1,2\}$. An element $X \in \mathcal{H}$ can be identified with a vector in $\mathbb{R}^{2}$. Let $\leq$ be the usual partial order of $\mathbb{R}^{2}$. The relevant claims are the positive ones, $\mathcal{R}=\mathcal{P}^{+}$.

There is a riskless asset $B$ and a risky asset $S$. At time zero, both assets have value $B_{0}=S_{0}=1$. The riskless asset yields $B_{1}=1+r$ for an interest rate $r>-1$ at time one, whereas the risky asset takes the values $u$ in state 1 and respectively $d$ in state 2 with $u>d$.

We use the riskless asset $B$ as numéraire. The discounted net return on the risky asset is $\hat{\ell}:=S_{1} /(1+r)-1$. I is the linear space spanned by $\hat{\ell}$. There is no arbitrage if and only if the unique candidate for a martingale probability of state one

$$
p^{*}=\frac{1+r-d}{u-d}
$$

belongs to $(0,1)$ which is equivalent to $u>1+r>d . p^{*}$ induces the unique martingale measure $\mathbb{P}^{*}$ with expectation

$$
\mathbb{E}^{*}[X]=p^{*} X(1)+\left(1-p^{*}\right) X_{1}(2) .
$$

$\mathbb{P}^{*}$ is a linear measure; moreover, it has the full support property since for every $R \in \mathcal{R}$ we have $\mathbb{E}^{*}[R]>0$. The market is viable with the single-agent economy $A=\left\{\complement^{*}\right\}$ where the preference relation $\preceq^{*}$ is given by the linear expectation $\mathbb{P}^{*}$, that is, $X \preceq^{*} Y$ if and only if $\mathbb{E}^{*}[X] \leq \mathbb{E}^{*}[Y]$. Indeed, under this preference $\ell \sim^{*} 0$ for any $\ell \in \mathcal{I}$ and $X \prec^{*} X+R$ for any $X \in \mathcal{H}$ and $R \in \mathcal{P}^{+}$. In particular, any $\ell \in \mathcal{I}$ is an optimal portfolio and the market is viable.

The preceding analysis carries over to all finite $\Omega$ and complete financial markets.

EXAMPLE 2.5-Ellsberg market: We illustrate the concepts used in our definition of viability and in Theorem 2.2 with the help of a market that is inspired by the Ellsberg thought experiments, the archetypal instances of ambiguity in economics and decision theory. In these experiments, an urn is called ambiguous if the exact composition is not known to the participants. Suppose for simplicity that we model an ambiguous urn that contains black and red balls; let it be known that the proportion of red balls is in the interval $\left[p_{*}, p^{*}\right] \subset[0,1]$. The finite state space is given by $\Omega:=\{$ red, black $\}$. The commodity space $\mathcal{H}=\mathbb{R}^{\Omega}$ is the set of functions on $\Omega$ with the usual order. Consider the claim that pays one dollar if a red ball is drawn, that is,

$$
X(\omega)= \begin{cases}1 & \text { if } \omega \text { is red, } \\ 0 & \text { if } \omega \text { is black. }\end{cases}
$$

In contrast to the frictionless atom of finance, we now suppose that due to ambiguity, the asset can be bought at price $p_{*}$ and sold at price $p^{*}>p_{*} \cdot{ }^{10}$ Then the set of net trades is given by positive cone (rather than a linear subspace) generated by $\ell_{1}(\omega)=X(\omega)-p^{*}$ and $\ell_{2}(\omega)=p_{*}-X(\omega)$, that is,

$$
\mathcal{I}=\left\{\lambda \ell_{1}+\mu \ell_{2}: \lambda, \mu \geq 0\right\} .
$$

\footnotetext{
${ }^{10}$ Such a price setting behavior is quite natural in today's regulated financial markets when banks take the model uncertainty or ambiguity of their algorithms into account and perform a number of stress tests to compute an interval of possible prices; compare the early account of such practices in Artzner et al. (1999).
} 
Let $\mathbb{P}_{p}$ be the measure that assigns probability $p$ to the event $\{$ red $\}$. The risk-neutral subjective expected utility agent's preference with this subjective belief $\mathbb{P}_{p}$ is denoted by $\preceq_{p}$. For this financial market, the set of absolutely continuous martingale expectations $\mathcal{Q}_{\mathrm{ac}}$ is given by

$$
\mathcal{Q}_{\mathrm{ac}}=\left\{\mathbb{P}_{p} ; p \in\left[p_{*}, p^{*}\right]\right\} \text {. }
$$

The set of agents $\mathcal{A}^{*}=\left\{\preceq_{p} ; p \in\left[p_{*}, p^{*}\right]\right\}$ supports this market in the sense of our viability definition. The corresponding sublinear martingale expectation is given by

$$
\mathcal{E}(\xi)=\max \left\{\mathbb{E}_{p_{*}}[\xi], \mathbb{E}_{p^{*}}[\xi]\right\} .
$$

In contrast to the frictionless, ambiguity-free, and complete atom of finance, we now have a continuum of heterogeneous risk-neutral agents $\mathcal{A}^{*}$ that support the market (instead of a unique risk-neutral agent). The model's ambiguity (or, the imprecise probabilistic information) is described by the sublinear pricing functional $\mathcal{E}(\xi)$. The corresponding ambiguity-averse Gilboa-Schmeidler agent's preferences are represented by the utility function

$$
U_{\mathrm{GS}}(\xi):=\inf _{p \in\left[p_{*}, p^{*}\right]} \mathbb{E}_{p}[\xi] .
$$

We have then $U_{\mathrm{GS}}(\ell) \leq 0$ for all $\ell \in \mathcal{I}$. When $0<p_{*}<p^{*}<1, U_{\mathrm{GS}}$ is strictly monotone and supports the market. Note however, that when $p_{*}=0$ or $p^{*}=1$, the agent with utility function $U_{\mathrm{GS}}$ does no longer support the market as strict monotonicity fails.

EXAMPLE 2.6-Multiple priors: Knightian uncertainty leads to frameworks in which single ambiguity-averse or expected utility maximizers might not suffice to support a given arbitrage-free market. ${ }^{11}$ Consider a one-step financial market that allows trading only at time zero. Suppose that the underlying financial market returns are too ambiguous or too nonstationary to be accurately estimated or modeled by a single probability distribution, yet agents are willing to take a stand on a range of possible models for returns. Such a situation of imprecise probabilistic information about the distribution of asset returns can be modeled by a set of priors $\mathcal{Q}$ on a measurable space $(\Omega, \mathcal{F})$. Let the common order be the quasi-sure order induced by the family of priors, that is,

$$
X \leq Y \quad \Leftrightarrow \quad \mathbb{Q}(X \leq Y)=1, \quad \forall \mathbb{Q} \in \mathcal{Q} .
$$

Then an event $A \subset \Omega$ is negligible 12 if $\mathbb{Q}(A)=0$ for every prior $\mathbb{Q} \in \mathcal{Q}$. The commodity space that represents all claims in this market is given by

$$
\mathcal{H}=\bigcap_{\mathbb{Q} \in \mathcal{Q}} \mathbb{L}^{2}(\Omega, \mathbb{Q}) .
$$

As the $\mathbb{L}^{2}$ spaces are defined as equivalence classes, two claims in $\mathcal{H}$ are in the same equivalence class if they differ only on a negligible set. Call a claim $R \geq 0$ relevant if the set $\{R>0\}$ is not negligible, that is, $\mathcal{R}=\mathcal{P}^{+}$. The set of net trades can be any convex cone included in

$$
\mathcal{I}_{0}=\left\{\ell \in \mathcal{H}: \mathbb{E}_{\mathbb{Q}}[\ell] \leq 0 \forall \mathbb{Q} \in \mathcal{Q}\right\} .
$$

\footnotetext{
${ }^{11}$ In a recent paper, Bartl (2019) analyzed portfolio and consumption choice in such discrete time models.

${ }^{12}$ These events are called polar in the mathematical literature on quasi-sure analysis.
} 
One might guess that the single Gilboa-Scheidler agent with the utility function

$$
U_{\mathrm{GS}}(X):=\inf _{\mathbb{Q} \in \mathcal{Q}} \mathbb{E}_{\mathbb{P}}[X], \quad X \in \mathcal{H}
$$

supports the financial market. However, this agent would support the financial market only when it satisfies the monotonicity condition (2.2), that is, $U_{\mathrm{GS}}(R)>0$ for every $R \in$ $\mathcal{R}$. This monotonicity implies that all priors must have the same null sets. Indeed, suppose that there are two priors $\mathbb{P}_{1}, \mathbb{P}_{2} \in \mathcal{Q}$ and an event $A$ satisfying $\mathbb{P}_{1}(A)>0=\mathbb{P}_{2}(A)$. Then $A$ is not negligible, and consequently, the claim $R=\chi_{A}$ is relevant. On the other hand, $U_{\mathrm{GS}}\left(\chi_{A}\right)=0$ showing that the Gilboa-Schmeidler agent is not strictly monotone when there are priors with different null-sets. ${ }^{13}$

Similarly, suppose that a single agent with a linear preference relation $\preceq_{\mathbb{Q}}$ given by the subjective expected utility function $U(X)=\mathbb{E}_{\mathbb{Q}}[X]$ for a prior $\mathbb{Q}$ supports the financial market. ${ }^{14}$ Let $A$ be a null set of $\mathbb{Q}$. Then this agent is indifferent between $\chi_{A}$ and the zero claim. Thus the monotonicity condition (2.2) implies that $\chi_{A}$ cannot be relevant and $A$ must be negligible. As negligible sets are null for every prior, we conclude that the null sets of $\mathbb{Q}$ and the negligible sets coincide.

We conclude that we need to reconsider the viability concept in models that do not allow to describe the negligible sets by a single probability measure. In particular, note that the family of heterogeneous agents $\left\{\preceq_{\mathbb{Q}}\right\}_{\mathbb{Q} \in \mathcal{Q}}$ with linear preferences induced by the set of priors $\mathcal{Q}$ supports the financial market.

EXAMPLE 2.7-Volatility uncertainty: In the classical Samuelson-Merton-BlackScholes model, the stock price is a geometric Brownian motion satisfying the stochastic differential equation $d S_{t}=\sigma S_{t} d B_{t}$ where $B$ is a standard Brownian motion and $\sigma$ is a positive constant modeling the volatility.

It is widely recognized and empirically well documented that volatility is time-varying and stochastic; a variety of complex stochastic models of the underlying dynamics have been proposed in turn. ${ }^{15}$ One might question whether it is plausible to assume that agents can identify one of these models uniquely, in particular when the relevant volatility is not directly observable. A robust modeling approach thus allows for a whole class of volatility models.

Let us consider a financial market with Knightian uncertainty about the volatility of the price process, as discussed in Epstein and Ji (2013) for consumption-based asset pricing and in Vorbrink (2014) for option pricing; any adapted process $\sigma$ taking values in a certain interval $[\underline{\sigma}, \bar{\sigma}]$ is a plausible volatility process. We denote this class by $\Sigma$; for a given $\sigma \in \Sigma$, we let $\mathbb{P}^{\sigma}$ be the corresponding distribution of the stock price process. The ambiguity about volatility is thus modeled by the the set of priors $\mathcal{M}=\left\{\mathbb{P}^{\sigma}: \sigma \in \Sigma\right\}$. Take $\mathcal{R}=\mathcal{P}^{+}$, and model the financial market by taking $\mathcal{I}$ to be the set of all stochastic integrals with simple integrands that are bounded from below. ${ }^{16}$

In this situation, priors in $\mathcal{M}$ are not mutually equivalent; in fact, typically they are singular to each other. A single probability space framework is not able to capture volatility

\footnotetext{
${ }^{13}$ Note that the same conclusion holds if we define $U_{\mathrm{GS}}$ with any set of probability measures equivalent to $\mathcal{Q}$.

${ }^{14} \mathrm{We}$ take a risk-neutral agent for simplicity. The argument holds as well for risk-averse agents with a standard Bernoulli utility function.

${ }^{15}$ Starting with the Heston model (Heston (1993)), a whole literature has explored ever more complex dynamics; see Ghysels, Harvey, and Renault (1996) for an overview.

${ }^{16}$ We refer the reader to Soner, Touzi, and Zhang $(2012,2013)$ for all the technical details, in particular, for the formal construction of $\left\{\mathbb{P}^{\sigma}: \sigma \in \Sigma\right\}$ and its subtle properties.
} 
uncertainty. In most finance models, the assumption of a reference probability was made for technical reasons, in order to be able to apply the Girsanov theorem. The restriction to a single probability space framework in diffusion models limits the set of possible models; only drift uncertainty can be captured by such models, not volatility uncertainty. The issue becomes even more pertinent if one wants to capture more complex financial models involving jumps.

As the negligible sets of $\mathcal{M}$ cannot be generated by any single probability measure, this financial market is not covered by the classical probability space framework. However, the family of heterogenous agents $\left\{\preceq_{\sigma}\right\}_{\sigma \in \Sigma}$ induced by the priors $\mathbb{P}^{\sigma}$ does support the financial market. Indeed, for every prior $\mathbb{P}^{\sigma}$, every $\ell \in \mathcal{I}$ is a $\mathbb{P}^{\sigma}$-local martingale, and consequently, $\mathbb{E}_{\mathbb{P} \sigma}[\ell] \leq 0$.

We now discuss briefly a variation of the example in which the set of relevant claims is derived from preferences. In a first step, let us consider agents who do not have a razor-sharp model of volatility, but are willing to use a robust version that is called " $\epsilon$ contamination" in statistics (Huber (1965)) and decision theory (Eichberger and Kelsey (1999)) For any reference volatility $\sigma \in \Sigma$ and contamination $\epsilon>0$, let

$$
\Sigma_{\sigma, \epsilon}:=\left\{\tilde{\sigma} \in \Sigma:\|\sigma-\tilde{\sigma}\|_{\infty} \leq \epsilon\right\}
$$

describe the sets of volatility models that are close to $\sigma$. The $\epsilon$-contamination preferences $\preceq_{\sigma, \epsilon}$ are represented by the utility functions

$$
U_{\sigma, \epsilon}(X):=\inf _{\sigma \in \Sigma_{\sigma, \epsilon}} \mathbb{E}_{\mathbb{P}^{\sigma}}[X] .
$$

We use these preferences to define the set of relevant claims as follows by setting

$$
\mathcal{R}=\left\{R \in \mathcal{P}_{+}: U_{\sigma, \epsilon}(R)>0 \text { for some } \sigma \in \Sigma, \epsilon>0\right\} .
$$

A payoff on an event is thus relevant if a bet on that event matters for some ambiguityaverse agent with $\epsilon$-contamination preferences. This technically more complex model is also included in our framework. Indeed, for $\sigma \in \Sigma$ and $\ell \in \mathcal{I}, \mathbb{E}_{\mathbb{P} \sigma}[\ell] \leq 0$, and thus $U_{\sigma, \epsilon}(\ell) \leq 0$, proving the optimality condition (2.1). The definition of $\mathcal{R}$ shows directly that the monotonicity condition (2.2) is satisfied by the family of heterogenous agents $\left\{\preceq_{\sigma, \epsilon}\right\}_{\{\sigma \in \Sigma, \epsilon>0\}}$.

\section{THE EFFICIENT MARKET HYPOTHESIS}

The Efficient Market Hypothesis (EMH) plays a fundamental role in the history of financial economics. Fama (1970) called markets informationally efficient if all available information is reflected properly in current asset prices. There are several interpretations of this conjecture; in the early days after its appearance, the efficient market hypothesis was usually interpreted as asset prices being random walks in the sense that (log-) returns be independent from the past and identically distributed with the mean return being equal to the return of a safe bond (Malkiel (2003)). Later, the informational efficiency of asset prices was interpreted as a martingale property; (conditional) expected returns of all assets are equal to the return of a safe bond under some probability measure. This conjecture of the financial market's being a "fair game" dates back to Bachelier (1900) and was rediscovered by Paul Samuelson (Samuelson $(1965,1973)$ ). In dynamic settings, market efficiency is thus strongly related to (publicly available) information. Under Knightian 
uncertainty, the role of information and the martingale property of prices needs to be adapted properly as we shall see in this section. ${ }^{17}$

Throughout this section, let us assume that we have a frictionless one-period or discrete-time multiple period financial market as in Example 4.1. In particular, the set of net trades $\mathcal{I}$ is a subspace of $\mathcal{H}$.

\subsection{A Strong Version of the Efficient Market Hypothesis Under Risk}

There are various ways to formalize the efficient market hypothesis. A particular strong interpretation of informational efficiency requires that expected returns of all risky investment be equal under a common prior. In our framework, such a conclusion results if the common order is derived from a common prior.

Let $\mathbb{P}$ be a probability measure on $(\Omega, \mathcal{F})$. Set $\mathcal{H}=\mathcal{L}^{1}(\Omega, \mathcal{F}, \mathbb{P})$. Let the common order be defined by saying $X \leq Y$ if and only if the expected payoffs under $\mathbb{P}$ satisfy

$$
\mathbb{E}_{\mathbb{P}}[X] \leq \mathbb{E}_{\mathbb{P}}[Y] .
$$

We call $\mathbb{P}$ the common prior of this model. In this case, negligible claims coincide with the claims with mean zero under $\mathbb{P}$. Moreover, $X \in \mathcal{P}$ if $\mathbb{E}_{\mathbb{P}}[X] \geq 0$. We take $\mathcal{R}=\mathcal{P}_{+}$.

PROPOSITION 3.1: Under the assumptions of this subsection, the financial market is viable if and only if the common prior $\mathbb{P}$ is a martingale measure. In this case, $\mathbb{P}$ is the unique martingale measure.

PROOF: Note that the common order as given by (3.1) is complete. If $\mathbb{P}$ is a martingale measure, the common order $\leq$ itself defines a linear preference relation under which the market is viable with $A=\{\leq\}$.

On the other hand if the market is viable, Theorem 2.2 ensure that there exists a sublinear martingale expectation with full support. By the Riesz duality theorem, a martingale functional $\phi \in \mathcal{Q}_{\mathrm{ac}}$ can be identified with a probability measure $\mathbb{Q}$ on $(\Omega, \mathcal{F})$. It is absolutely continuous (in our sense defined above) if and only if it assigns the value 0 to all negligible claims. As a consequence, we have $E_{\mathbb{Q}}[X]=0$ whenever $E_{\mathbb{P}}[X]=0$. Then $\mathbb{Q}=\mathbb{P}$ follows. ${ }^{18}$

The only absolutely continuous martingale measure is the common prior itself. As a consequence, all traded assets have zero net expected return under the common prior. A financial market is thus viable if and only if the strong form of the expectations hypothesis holds true.

\subsection{A Weak Version of the Efficient Market Hypothesis Under Risk}

A weaker version of the efficient market hypothesis states that expected returns are equal under some (pricing) probability measure $\mathbb{P}^{*}$ that is equivalent to the common prior (or "real world" probability) $\mathbb{P}$.

\footnotetext{
${ }^{17}$ We refer to Jarrow and Larsson (2012) for a detailed analysis of the interplay between different information sets and market efficiency under a common prior. In our framework, the information flow is taken as given; it is implicitly encoded in the set of available claims $\mathcal{I}$. We do not consider the issue of private information of insiders.

${ }^{18}$ If $\mathbb{Q} \neq \mathbb{P}$, there is an event $A \in \mathcal{F}$ with $\mathbb{Q}(A)<\mathbb{P}(A)$. Set $X=1_{A}-\mathbb{P}(A)$. Then $0=E_{\mathbb{P}}[X]>\mathbb{Q}(A)-$ $\mathbb{P}(A)=E_{\mathbb{Q}}[X]$.
} 
Let $\mathbb{P}$ be a probability on $(\Omega, \mathcal{F})$ and $\mathcal{H}=\mathcal{L}^{1}(\Omega, \mathcal{F}, \mathbb{P})$. In this example, the common order is given by the almost sure order under the common prior $\mathbb{P}$, that is,

$$
X \leq Y \quad \Leftrightarrow \quad \mathbb{P}(X \leq Y)=1 .
$$

A payoff is negligible if it vanishes $\mathbb{P}$-almost surely and is positive if it is $\mathbb{P}$-almost surely nonnegative. Let the relevant claims $\mathcal{R}$ consist of the $\mathbb{P}$-almost surely nonnegative payoffs that are strictly positive with positive $\mathbb{P}$-probability,

$$
\mathcal{R}=\left\{R \in \mathcal{L}^{1}(\Omega, \mathcal{F}, \mathbb{P})_{+}: \mathbb{P}(R>0)>0\right\} .
$$

A functional $\phi \in \mathcal{H}_{+}^{\prime}$ is an absolutely continuous martingale functional if and only if it can be identified with a probability measure $\mathbb{Q}$ that is absolutely continuous with respect to $\mathbb{P}$ and if all net trades have expectation zero under $\phi$. In other words, discounted asset prices are $\mathbb{Q}$-martingales. We thus obtain a version of the fundamental theorem of asset pricing under risk, similar to Harrison and Kreps (1979) and Dalang, Morton, and Willinger (1990).

PROPOSITION 3.2: Under the assumptions of this subsection, the financial market is viable if and only if there is a martingale measure $\mathbb{Q}$ that has a bounded density with respect to $\mathbb{P}$.

Proof: If $\mathbb{Q}$ is a martingale measure equivalent to $\mathbb{P}$, define $X \preceq^{*} Y$ if and only if $\mathbb{E}_{\mathbb{Q}}[X] \leq \mathbb{E}_{\mathbb{Q}}[Y]$. Then the market is viable with $A=\left\{\preceq^{*}\right\}$. Condition (2.2) is satisfied because $\mathbb{Q}$ is equivalent to $\mathbb{P}$.

If the market is viable, Theorem 2.2 ensures that there exists a sublinear martingale expectation with full support. By the Riesz duality theorem, a martingale functional $\phi \in \mathcal{Q}_{\mathrm{ac}}$ can be identified with a probability measure $\mathbb{Q}_{\phi}$ that is absolutely continuous with respect to $\mathbb{P}$, has a bounded density with respect to $\mathbb{P}$, and all net trades have zero expectation zero under $\mathbb{Q}_{\phi}$. In other words, discounted asset prices are $\mathbb{Q}_{\phi}$-martingales. From the full support property, the family $\left\{\mathbb{Q}_{\phi}\right\}_{\phi \in \mathcal{Q}_{\mathrm{ac}}}$ is equivalent to $\mathbb{P}$, meaning that $\mathbb{Q}_{\phi}(A)=0$ for every $\phi \in \mathcal{Q}_{\mathrm{ac}}$ if and only if $\mathbb{P}(A)=0$. By the Halmos-Savage theorem (Halmos and Savage (1949), (Föllmer and Schied, 2011, Theorem 1.61)), there exists a countable subfamily $\left\{\mathbb{Q}_{\phi_{n}}\right\}_{n \in \mathbb{N}} \subset\left\{\mathbb{Q}_{\phi}\right\}_{\phi \in \mathcal{Q}_{a c}}$, which is equivalent to $\mathbb{P}$. The measure $\mathbb{Q}:=\sum_{n=1}^{\infty} 2^{-n} \mathbb{Q}_{\phi_{n}}$ is the desired equivalent martingale measure.

Q.E.D.

\subsection{The Efficient Market Hypothesis Under Knightian Uncertainty}

We turn our attention to the EMH under Knightian uncertainty. We consider first the case when the common order is derived from a common set of priors, inspired by the multiple prior approach in decision theory (Bewley (2002), Gilboa and Schmeidler (1989)). We then discuss a second-order Bayesian approach that is inspired by the smooth ambiguity model (Klibanoff, Marinacci, and Mukerji (2005)).

\subsubsection{A Strong Version Under Knightian Uncertainty}

We consider a generalization of the original EMH to Knightian uncertainty that shares a certain analogy with Bewley's incomplete expected utility model (Bewley (2002)) and Gilboa and Schmeidler's maxmin expected utility (Gilboa and Schmeidler (1989)). ${ }^{19}$

\footnotetext{
${ }^{19}$ For the relation between the two approaches, compare also the discussion of objective and subjective ambiguity in Gilboa, Maccheroni, Marinacci, and Schmeidler (2010).
} 
Let $\Omega$ be a metric space with metric $d$ and Borel sets $\mathcal{F}$. Let $\mathcal{M}$ be a convex, weak* closed set of priors on $(\Omega, \mathcal{F})$. Define the seminorm

$$
\|X\|_{\mathcal{M}}:=\sup _{\mathbb{P} \in \mathcal{M}} \mathbb{E}_{\mathbb{P}}|X|
$$

Let $\mathcal{L}^{1}(\Omega, \mathcal{F}, \mathcal{M})$ be the closure of continuous and bounded functions on $\Omega$ under the seminorm $\|\cdot\|_{\mathcal{M}}$. If we identify the functions which are $\mathbb{P}$-almost surely equal for every $\mathbb{P} \in \mathcal{M}$, then $\mathcal{H}=\mathcal{L}^{1}(\Omega, \mathcal{F}, \mathcal{M})$ is a Banach space. The topological dual of $\mathcal{L}^{1}(\Omega, \mathcal{F}, \mathcal{M})$ can be identified with probability measures that admit a bounded density with respect to some measure in $\mathcal{M}$ (Bion-Nadal, Kervarec et al. (2012), Beissner and Denis (2018)). Therefore, any absolutely continuous martingale functional $\mathbb{Q} \in \mathcal{Q}_{\text {ac }}$ is a probability measure and $\mathcal{M}$ is closed in the weak ${ }^{*}$ topology induced by $\mathcal{L}^{1}(\Omega, \mathcal{F}, \mathcal{M})$.

Consider the uniform order induced by expectations over $\mathcal{M}$,

$$
X \leq Y \quad \Leftrightarrow \quad \forall \mathbb{P} \in \mathcal{M} \quad \mathbb{E}_{\mathbb{P}}[X] \leq \mathbb{E}_{\mathbb{P}}[Y] .
$$

Then $Z \in \mathcal{Z}$ if $\mathbb{E}_{\mathbb{P}}[Z]=0$ for every $\mathbb{P} \in \mathcal{M}$. A claim $X$ is nonnegative if $\mathbb{E}_{\mathbb{P}}[X] \geq 0$ for every $\mathbb{P} \in \mathcal{M}$. Let the relevant claims consist of nonnegative claims with a positive return under some prior belief, that is,

$$
\mathcal{R}=\left\{R \in \mathcal{H}: 0 \leq \inf _{\mathbb{P} \in \mathcal{M}} \mathbb{E}_{\mathbb{P}}[R] \text { and } 0<\sup _{\mathbb{P} \in \mathcal{M}} \mathbb{E}_{\mathbb{P}}[R]\right\}
$$

PROPOSITION 3.3: Under the assumptions of this subsection, if the financial market is viable, then the set of absolutely continuous martingale functionals $\mathcal{Q}_{\mathrm{ac}}$ is a subset of the set of priors $\mathcal{M}$.

Proof: Set $\mathcal{E}_{\mathcal{M}}(X):=\sup _{\mathbb{P} \in \mathcal{M}} \mathbb{E}_{\mathbb{P}}[X]$. Then $Y \leq 0$ if and only if $\mathcal{E}_{\mathcal{M}}(Y) \leq 0$. Fix $\mathbb{Q} \in$ $\mathcal{Q}_{\text {ac }}$ with the preference relation given by $X \preceq_{\mathbb{Q}} Y$ if $\mathbb{E}_{\mathbb{Q}}[X-Y] \leq 0$.

Let us assume that $\mathbb{Q} \notin \mathcal{M}$. Since $\mathcal{M}$ is a weak ${ }^{*}$-closed and convex subset of the topological dual of $\mathcal{L}^{1}(\Omega, \mathcal{F}, \mathcal{M})$, there exists $X^{*} \in \mathcal{L}^{1}(\Omega, \mathcal{F}, \mathcal{M})$ with $\mathcal{E}_{\mathcal{M}}\left(X^{*}\right)<0<\mathbb{E}_{\mathbb{Q}}\left[X^{*}\right]$ by the Hahn-Banach theorem. In particular, $X^{*} \in \mathcal{L}^{1}(\Omega, \mathcal{F}, \mathcal{M})$ and $X^{*} \leq 0$. Since $\preceq_{\mathbb{Q}}$ is weakly monotone with respect to $\leq, X^{*} \preceq_{\mathbb{Q}} 0$. Hence, $\mathbb{E}_{\mathbb{Q}}\left[X^{*}\right] \leq 0$ contradicting the choice of $X^{*}$. Therefore, $\mathcal{Q}_{\text {ac }} \subset \mathcal{M}$.

Q.E.D.

Expected returns of traded securities are thus not necessarily the same under all $\mathbb{P} \in \mathcal{M}$. However, the set of martingale measures is a subset of $\mathcal{M}$ here, and thus the strong form of the EMH holds true on a subset of the set of priors $\mathcal{M}$.

In general, it is not possible to characterize the set of martingale measures in more detail. However, we can identify a subspace of claims on which expectations under all priors coincide. Let $\mathcal{H}_{\mathcal{M}}$ be the subspace of claims that have no ambiguity in the mean in the sense that $\mathbb{E}_{\mathbb{P}}[X]$ is the same constant for all $\mathbb{P} \in \mathcal{M}$. Consider the submarket $\left(\mathcal{H}_{\mathcal{M}}, \tau, \leq, \mathcal{I}_{\mathcal{M}}, \mathcal{R}_{\mathcal{M}}\right)$ with $\mathcal{I}_{\mathcal{M}}:=\mathcal{I} \cap \mathcal{H}_{\mathcal{M}}$ and $\mathcal{R}_{\mathcal{M}}:=\mathcal{R} \cap \mathcal{H}_{\mathcal{M}}$. Restricted to this market, the sets of measures $\mathcal{Q}_{\text {ac }}$ and $\mathcal{M}$ are identical and the strong EMH holds true.

The following simple example illustrates these points.

EXAMPLE 3.4: Let $\Omega=\{0,1\}^{2}, \mathcal{H}$ be all functions on $\Omega$. Then, $\mathcal{H}=\mathbb{R}^{4}$ and we write $X=(x, y, v, w)$ for any $X \in \mathcal{H}$. Let $\mathcal{I}=\{(x, y, 0,0): x+y=0\}$. Consider the priors given by

$$
\mathcal{M}:=\left\{\left(p, \frac{1}{2}-p, \frac{1}{4}, \frac{1}{4}\right): p \in\left[\frac{1}{6}, \frac{1}{3}\right]\right\} .
$$


There is Knightian uncertainty about the first two states, yet no Knightian uncertainty about the last two states.

A claim $Z=\left(Z_{1}, Z_{2}, Z_{3}, Z_{4}\right)$ is negligible iff $Z_{1}=Z_{2}$ and $Z_{4}=-2 Z_{1}-Z_{3}$. In particular, $X=(1,1,0,-2)$ and $Y=(0,0,1,-1)$ are negligible. Now let $\mathbb{Q}^{*}=\left(q_{1}, q_{2}, q_{3}, q_{4}\right) \in \mathcal{Q}_{\mathrm{ac}}$. The martingale property implies $q_{1}=q_{2}$. Absolute continuity requires that $\mathbb{E}_{\mathbb{Q}^{*}}[X]=0$ and $\mathbb{E}_{\mathbb{Q}^{*}}[Y]=0$, or $q_{1}+q_{2}-2 q_{4}=0$ and $q_{3}=q_{4}$. From here, we obtain with $q_{1}=q_{2}$ that $q_{1}=q_{2}=q_{3}=q_{4}$, so $\mathbb{Q}^{*}=\left(\frac{1}{4}, \frac{1}{4}, \frac{1}{4}, \frac{1}{4}\right)$. Notice that $\mathbb{Q}^{*} \in \mathcal{M}$.

In this case, $\mathcal{H}_{\mathcal{M}}=\{X=(x, y, v, w) \in \mathcal{H}: x=y\}$. In particular, all priors in $\mathcal{M}$ coincide with $\mathbb{Q}^{*}$ when restricted to $\mathcal{H}_{\mathcal{M}}$. Hence, for the claims that are mean-ambiguity-free, the strong efficient market hypothesis holds true.

\subsubsection{A Weak Version Under Knightian Uncertainty}

Let $\mathcal{M}$ be a common set of priors on $(\Omega, \mathcal{F})$. Let $\mathcal{H}$ be the space of bounded, measurable functions. Let the common order be given by the quasi-sure ordering under the common set of priors $\mathcal{M}$, that is,

$$
X \leq Y \quad \Leftrightarrow \quad \mathbb{P}(X \leq Y)=1, \quad \forall \mathbb{P} \in \mathcal{M} .
$$

In this case, a claim $X$ is negligible if it vanishes $\mathcal{M}$-quasi-surely, that is, with probability one for all $\mathbb{P} \in \mathcal{M}$. An indicator function $1_{A}$ is thus negligible if the set $A$ is polar, that is, a null set with respect to every probability in $\mathcal{M}$. Take the set of relevant claims to be ${ }^{20}$

$$
\mathcal{R}=\{R \in \mathcal{P}: \exists \mathbb{P} \in \mathcal{M} \text { such that } \mathbb{P}(R>0)>0\} .
$$

PROPOSITION 3.5: Under the assumptions of this subsection, the financial market is viable if and only if there is a set of finitely additive martingale measures $\mathcal{Q}$ that has the same polar sets as the common set of priors $\mathcal{M}$.

PROOF: Suppose that the market is viable. We show that the class $\mathcal{Q}_{\text {ac }}$ from Theorem 2.2 satisfies the desired properties. The martingale property follows by definition and from the fact that $\mathcal{I}$ is a linear space. Suppose that $A$ is polar. Then $1_{A}$ is negligible and from the absolute continuity property, it follows $\phi(A)=0$ for any $\phi \in \mathcal{Q}_{\text {ac. }}$ On the other hand, if $A$ is not polar, $1_{A} \in \mathcal{R}$ and from the full support property, it follows that there exists $\phi_{A} \in \mathcal{Q}_{\mathrm{ac}}$ such that $\phi_{A}(A)>0$. Thus, $A$ is not $\mathcal{Q}_{\mathrm{ac}}$-polar. We conclude that $\mathcal{M}$ and $\mathcal{Q}_{\text {ac }}$ share the same polar sets. For the converse implication, define $\mathcal{E}(\cdot):=\sup _{\phi \in \mathcal{Q}} \mathbb{E}_{\phi}[\cdot]$. Using the same argument as above, $\mathcal{E}$ is a sublinear martingale expectation with full support. From Theorem 2.2, the market is viable.

Q.E.D.

Under Knightian uncertainty, there can be indeterminacy in arbitrage-free prices as there is frequently a range of economically justifiable arbitrage-free prices. Such indeterminacy has been observed in full general equilibrium analysis as well (Rigotti and Shannon (2005), Dana and Riedel (2013), Beissner and Riedel (2019)). In this sense, Knightian uncertainty shares a similarity with incomplete markets and other frictions like transaction costs, but the economic reason for the indeterminacy is different.

\footnotetext{
${ }^{20}$ These sets of positive and relevant claims can be derived from Gilboa-Schmeidler utilities. Define $X \preceq Y$ if and only if $\inf _{\mathbb{P} \in \mathcal{M}} E_{\mathbb{P}}[U(X)] \leq \inf _{\mathbb{P} \in \mathcal{M}} E_{\mathbb{P}}[U(Y)]$ for all strictly increasing and concave real functions $U$. Then $0 \preceq Y$ implies that for all priors $\mathbb{P} \in \mathcal{M}$, every risk-averse expected utility agent prefers $Y$ to the null claim. As is well known, this is equivalent to $Y$ dominating the null claim in the sense of second-order stochastic dominance for every $\mathbb{P} \in \mathcal{M}$, implying that $Y$ is nonnegative almost surely for all $\mathbb{P} \in \mathcal{M}$.
} 


\subsubsection{A Second-Order Bayesian Version of the Efficient Market Hypothesis}

We now consider a common order $\leq$ obtained by a second-order Bayesian approach, in the spirit of the smooth ambiguity model (Klibanoff, Marinacci, and Mukerji (2005)).

Let $\mathcal{F}$ be a sigma algebra on $\Omega$ and $\mathfrak{P}=\mathfrak{P}(\Omega)$ the set of all probability measures on $(\Omega, \mathcal{F})$. Let $\mu$ be a second-order prior, that is, a probability measure ${ }^{21}$ on $\mathfrak{P}$. The common prior in this setting is given by the probability measure $\hat{\mathbb{P}}: \mathcal{F} \rightarrow[0,1]$ defined as $\hat{\mathbb{P}}(A)=\int_{\mathfrak{P}} \mathbb{P}(A) \mu(d \mathbb{P})$. Let $\mathcal{H}=\mathcal{L}^{1}(\Omega, \mathcal{F}, \hat{\mathbb{P}})$.

The common order is given by

$$
X \leq Y \quad \Leftrightarrow \quad \mu(\{\mathbb{P} \in \mathfrak{P}: \mathbb{P}(X \leq Y)=1\})=1 .
$$

A claim is positive if it is $\mathbb{P}$-almost surely nonnegative for all priors in the support of the second-order prior $\mu$. A claim is relevant if the set of beliefs $\mathbb{P}$ under which the claim is strictly positive with positive probability is not negligible according to the second-order prior. $^{22}$

PROPOSITION 3.6: Under the assumptions of this subsection, the financial market is viable if and only if there is a martingale measure $\mathbb{Q}$ that has the form

$$
\mathbb{Q}(A)=\int_{\mathfrak{P}} \int_{A} D d \mathbb{P} \mu(d \mathbb{P})
$$

for some state price density $D$.

Proof: The set function $\hat{\mathbb{P}}: \mathcal{F} \rightarrow[0,1]$ defined as $\hat{\mathbb{P}}(A)=\int_{\mathfrak{P}} \mathbb{P}(A) \mu(d \mathbb{P})$ is a probability measure on $(\Omega, \mathcal{F})$. The induced $\hat{\mathbb{P}}$-a.s. order coincides with $\leq$ of this subsection. The result thus follows from Proposition 3.2 and the rules of integration with respect to $\hat{\mathbb{P}}$.

The smooth ambiguity model thus leads to a second-order Bayesian approach for asset returns. All asset returns are equal to the safe return for some second-order martingale measure; the expectation is the average expected return corresponding to a risk-neutral second-order prior $\mathbb{Q}$.

\section{FURTHER DISCUSSION OF THE MODELING PHILOSOPHY}

This section discusses various aspects of our modeling approach in more detail. We first discuss the motivation of using a common order instead of a probabilistic framework.

\footnotetext{
${ }^{21}$ From Theorem 15.18 of Aliprantis and Border (1999), the space of probability measure is a Borel space if and only if $\Omega$ is a Borel space. This allows to define second-order priors.

${ }^{22}$ The order used in this section can be derived from smooth ambiguity utility functions. Define $X \leq Y$ if and only if

$$
\int_{\mathfrak{P}} \psi\left(\mathbb{E}_{\mathbb{P}}[U(X)]\right) \mu(d \mathbb{P}) \leq \int_{\mathfrak{P}} \psi\left(\mathbb{E}_{\mathbb{P}}[U(X)]\right) \mu(d \mathbb{P})
$$

for all strictly increasing and concave real functions $U$ and $\psi$. Recall that $\psi$ reflects uncertainty aversion. Then $0 \leq Y$ is equivalent to $Y$ dominating the zero claim in the sense of second-order stochastic dominance for $\mu$-almost all $\mathbb{P} \in \mathfrak{P}$.
} 
We then explain how various static and dynamic financial markets are embedded in our abstract model. Our notion of viability is related to the usual notion of equilibrium of a competitive market with heterogeneous agents. We discuss the role of sublinear versus linear prices in our theory, and lastly show how our concept of relevant claims can be used to unify various notions of arbitrage in the finance literature.

A Common Order versus a Common Probabilistic Framework. Preference properties shared by all agents in the market will be reflected in equilibrium prices.

A situation of risk is described by the fact that agents share a common prior; in the laboratory, a random experiment based on an objective device like a roulette wheel or a coin toss simulates such a market environment. If no such objective device can be invoked, as in the real world, one might still presume the existence of a common subjective belief for all market participants as it is done (implicitly) in the capital asset pricing model as well as in its its consumption-based version. Such an assumption might be too strong; the Ellsberg experiments show how to create an environment of Knightian uncertainty in the lab. In complex financial markets in which credit risk claims, options on term structure shapes and volatility dynamics are traded, Knightian uncertainty plays a prominent role because agents lack precise probability estimates of crucial model parameters and they might be wary of potential structural breaks in the data. Moreover, as Epstein and Ji (2013) have shown (see also Example 2.7), if we model Knightian uncertainty about volatility, it is logically impossible to construct a reference probability measure.

We take these considerations as a motivation to forego any explicit or implicit probabilistic assumption, be it a common prior $\mathbb{P}$ or the weaker assumption of a common reference probability. Instead, we base our analysis on a common order $\leq$, a far weaker assumption that only requires a (typically incomplete) unanimous dominance criterion. A minimal example of a common order is the pointwise order. Pointwise dominance is certainly a criterion that we might assume to be unanimously shared in the context of monetary or single good payoffs. The generality of our approach allows to cover a wide variety of situations, including the well-studied case of risk as well as situations of Knightian uncertainty as we have seen in the previous section. ${ }^{23}$

The Financial Market. We model the financial market in a rather reduced form with the help of the convex cone $\mathcal{I}$. This abstract approach is sufficient for our purpose of discussing the relation of arbitrage and viability. In the next example, we show how the usual models of static and dynamic trading are embedded.

\footnotetext{
${ }^{23}$ It might be interesting to note an alternative way of setting up the model in which the common order is derived from a class of given preference relations. Suppose that no common order is a priori given. Instead, we start with a class of preference relations $\mathcal{A}_{0}$ on the commodity space $\mathcal{H}$ that are convex and $\tau$-lower semicontinuous. We can then define the uniform order derived from the set of preference relations $\mathcal{A}_{0}$ as follows. Let

$$
\mathcal{Z}_{\preceq}:=\{Z \in \mathcal{H}: X \preceq Z+X \preceq X, \forall X \in \mathcal{H}\},
$$

be the set of negligible (or null) claims for the preference relation $\preceq \in \mathcal{A}_{0}$. We call $\mathcal{Z}_{\text {uni }}:=\bigcap_{\leq \in \mathcal{A}_{0}} \mathcal{Z}_{\leq}$the set of unanimously negligible claims. Let the uniform pre-order $\leq_{\text {uni }}$ on $\mathcal{H}$ be given by $X \leq_{\text {uni }} Y$ if and only if there exists $Z \in \mathcal{Z}_{\text {uni }}$ such that $X(\omega) \leq Y(\omega)+Z(\omega)$ for all $\omega \in \Omega$. Note that we use the pointwise order on the reals and the set of uniformly negligible payoffs to derive the common order from $\mathcal{A}_{0} .\left(\mathcal{H}, \leq_{\text {uni }}\right)$ is then a preordered vector space, and agents' preferences in $\mathcal{A}_{0}$ are monotone with respect to the uniform preorder. Let $\mathcal{A}$ be the set of (convex, etc.) preferences that are monotone with respect to the common order. Note that $\mathcal{A}$ contains $\mathcal{A}_{0}$, but is usually larger than $\mathcal{A}_{0}$.
} 
EXAMPLE 4.1: We consider four markets with increasing complexity.

1. In a one period setting with finitely many states $\Omega=\{1, \ldots, N\}$, a financial market with $J+1$ securities can be described by its initial prices $x_{j} \geq 0, j=0, \ldots, J$ and a $(J+1) \times$ $N$-payoff matrix $F$, compare LeRoy and Werner (2014). A portfolio $\bar{H}=\left(H_{0}, \ldots, H_{J}\right) \in$ $\mathbb{R}^{J+1}$ has the payoff $\bar{H} F=\left(\sum_{j=0}^{J} H_{j} F_{j \omega}\right)_{\omega=1, \ldots, N}$; its initial cost satisfies $H \cdot x=\sum_{j=0}^{J} H_{j} x_{j}$. If the zeroth asset is riskless with a price $x_{0}=1$ and pays off 1 in all states of the world, then a net trade with zero initial cost can be expressed in terms of the portfolio of risky assets $H=\left(H_{1}, \ldots, H_{J}\right) \in \mathbb{R}^{J}$ and the return matrix $\hat{F}=\left(F_{j \omega}-x_{j}\right)_{j=1, \ldots, J, \omega=1, \ldots, N} . \mathcal{I}$ is given by the image of the $J \times N$ return matrix $\hat{F}$, that is,

$$
\mathcal{I}=\left\{H \hat{F}: H \in \mathbb{R}^{J}\right\}
$$

2. Our model includes the case of finitely many trading periods. Let $\mathbb{F}:=\left(\mathcal{F}_{t}\right)_{t=0}^{T}$ be a filtration on $(\Omega, \mathcal{F})$ and $S=\left(S_{t}\right)_{t=0}^{T}$ be an adapted stochastic process with values in $\mathbb{R}_{+}^{J}$ for some $J \geq 1 ; S$ models the uncertain assets. We assume that a riskless bond with interest rate zero is also given. Then the set of net trades can be described by the gains from trade processes: $\ell \in \mathcal{H}$ is in $\mathcal{I}$ provided that there exists predictable integrands $H_{t} \in$ $\left(\mathcal{L}^{0}\left(\Omega, \mathcal{F}_{t-1}\right)\right)^{J}$ for $t=1, \ldots, T$ such that

$$
\ell=(H \cdot S)_{T}:=\sum_{t=1}^{T} H_{t} \cdot \Delta S_{t}, \quad \text { where } \Delta S_{t}:=\left(S_{t}-S_{t-1}\right) .
$$

In the frictionless case, the set of net trades is a subspace of $\mathcal{H}$. In general, one might impose restrictions on the set of admissible trading strategies. For example, one might exclude short-selling of risky assets, or impose a bound on agents' credit line; in these cases, the marketed subspace $\mathcal{I}$ is a convex cone, compare Luttmer (1996), Jouini and Kallal (1995), and Araujo, Chateauneuf, and Faro (2018), for example,

3. In Harrison and Kreps (1979), the market is described by a marketed space $M \subset$ $\mathcal{L}^{2}(\Omega, \mathcal{F}, \mathbb{P})$ and a (continuous) linear functional $\pi$ on $M$. In this case, $\mathcal{I}$ is the kernel of the price system, that is,

$$
\mathcal{I}=\{X \in M: \pi(X)=0\} .
$$

4. In continuous time, the set of net trades consists of stochastic integrals of the form

$$
\mathcal{I}=\left\{\int_{0}^{T} \theta_{u} \cdot d S_{u}: \theta \in \mathcal{A}_{\mathrm{adm}}\right\}
$$

for a suitable set of admissible strategies $\mathcal{A}_{\mathrm{adm}}$. There are several possible choices of such a set. When the stock price process $S$ is a semimartingale one example of $\mathcal{A}_{\text {adm }}$ is the set of all $S$-integrable, predictable processes whose integral is bounded from below. ${ }^{24}$ Other typical choices for $\mathcal{A}_{\text {adm }}$ would consist of simple integrands only; when $S$ is a continuous process and $\mathcal{A}_{\mathrm{adm}}$ is the set of process with finite variation then the above integral can be defined through integration by parts (see Dolinsky and Soner (2014a, 2015)).

In general, the absence of a common prior poses some nontrivial technical questions about the integrability of contingent claims and net trades. Clearly, it is possible to restrict

\footnotetext{
${ }^{24}$ In continuous time, to avoid doubling strategies a lower bound (maybe more general than above) has to be imposed on the stochastic integrals. In such cases, the set $\mathcal{I}$ is not a linear space.
} 
the commodity space to the class of bounded measurable functions (that are integrable with respect to any prior). The condition $\mathcal{I} \subset \mathcal{H}$ could be restrictive in some applications and we provide a way to overcome this difficulty in the supplementary online material.

Viability. In Harrison and Kreps (1979), Kreps (1981), viability is defined with the help of a single representative agent with a strictly monotone preference relation. We allow for many agents, with weakly monotone preferences, yet the family as a whole satisfies a strict monotonicity condition (equation (2.2)). We show that one can prove the same results as Harrison and Kreps in classic cases, and one obtains easily the equivalence result in new cases involving Knightian uncertainty as in the Epstein-Ji model (Example 2.7). From the economic point of view, our definition of viability does not contradict the intuitive idea of an economic equilibrium because that idea does allow for many agents and weakly monotone preferences, of course. We thus believe that the use of our new definition is justified (and its usefulness is illustrated by the proofs of our abstract theorems).

The reader might note that our notion of equilibrium does not model endowments explicitly as we assume that the zero trade is optimal for each agent. This reduced approach comes without loss of generality in our context. In general, an agent is given by a preference relation $\succeq \in \mathcal{A}$ and an endowment $e \in \mathcal{H}$. Given the set of net trades, the agent chooses $\ell^{*} \in \mathcal{I}$ such that $e+\ell^{*} \succeq e+\ell$ for all $\ell \in \mathcal{I}$. By suitably modifying the preference relation, this can be reduced to the optimality of the zero trade at the zero endowment for a suitably modified preference relation. Let $X \succeq^{\prime} Y$ if and only if $X+e+\ell^{*} \succeq Y+e+\ell^{*}$. It is easy to check that $\succeq^{\prime}$ is also an admissible preference relation. For the new preference relation $\succeq^{\prime}$, we then have $0 \succeq^{\prime} \ell$ if and only if $e+\ell^{*} \succeq e+\ell^{*}+\ell$. As $\mathcal{I}$ is a cone, $\ell+\ell^{*} \in \mathcal{I}$, and we conclude that we have indeed $0 \succeq^{\prime} \ell$ for all $\ell \in \mathcal{I}$.

Sublinear Expectations. Our fundamental theorem of asset pricing characterizes the absence of arbitrage with the help of a nonadditive expectation $\mathcal{E}$. In decision theory, non-additive probabilities have a long history; Schmeidler (1989) introduces an extension of expected utility theory based on nonadditive probabilities. The widely used max-min expected utility model of Gilboa and Schmeidler (1989) is another instance. If we define the subjective expectation of a payoff to be the minimal expected payoff over a class of priors, then the resulting notion of expectation has some of the common properties of an expectation like monotonicity and preservation of constants, but is no longer additive.

In our case, the nonadditive expectation has a more objective than subjective flavor because it describes the pricing functional of the market. It assigns a nonpositive value to all net trades; in this sense, net trades have the (super)martingale property under this expectation. If we assume for the sake of the discussion that the set of net trades is a linear subspace, then the pricing functional has to be additive over that subspace. As a consequence, the value of all net trades under the sublinear pricing expectation is zero. For contingent claims that lie outside the marketed subspace, the pricing operation of the market is subadditive.

Whereas an additive probability measure is sufficient to characterize viable markets in probabilistic models, under uncertainty, it is more sensible to consider nonadditive notion of expectation ${ }^{25}$ as, for example, in the framework of Example 2.5 because it allows to characterize fully the ambiguity of market prices. ${ }^{26}$

\footnotetext{
${ }^{25}$ Beissner and Riedel (2019) developed a general equilibrium model based on such nonadditive pricing functionals.

${ }^{26}$ In some technically complex models, only sublinear functionals can be strictly positive.
} 
Relevant Claims. We use the notion of relevant claims to generalize the typical approach to define arbitrage as positive net trades. This approach introduces some additional flexibility and allows to cover variants of the notion of arbitrage that were discussed in the literature. For example, if some positive claim cannot be liquidated without costs, agents would not consider a net trade that achieves such a payoff as free lunch if the liquidation costs are larger than the potential gains. It is then reasonable to consider as relevant only a restricted class of positive claims, possibly only cash. Moreover, relevant payoffs identify those nonnegative consumption plans that some market participants strictly prefer to the null plan. The commodity spaces that are used to model markets with Knightian uncertainty may be quite large, for example, when we work with the space of all bounded, measurable functions. In such models, it makes sense to work with a set of relevant claims that is smaller than the cone of positive claims, see also Example 2.7.

We illustrate the usefulness of the notion of relevant claims by relating our work to recent results in mathematical finance. Our approach gives a microeconomic foundation to the characterization of absence of arbitrage in "robust" or "model-free" finance.

Let $\Omega$ be a metric space with the pointwise order $\leq$. In the finance literature, this approach is called model-independent as it does not rely on any probability measure. There is still a model, of course, given by $\Omega$ and the pointwise order.

A claim is nonnegative, $X \in \mathcal{P}$, if $X(\omega) \geq 0$ for every $\omega \in \Omega$ and $R \in \mathcal{P}^{+}$if $R \in \mathcal{P}$ and there exists $\omega_{0} \in \Omega$ such that $R\left(\omega_{0}\right)>0$.

In the literature, several different notions of arbitrage have been used. Our framework allows to unify these different approaches under one framework with the help of the notion of relevant claims. ${ }^{27}$ We start with the following large set of relevant claims

$$
\mathcal{R}_{\text {op }}:=\mathcal{P}^{+}=\left\{R \in \mathcal{P}: \exists \omega_{0} \in \Omega \text { such that } R\left(\omega_{0}\right)>0\right\} .
$$

With this notion of relevance, an investment opportunity $\ell$ is an arbitrage if $\ell(\omega) \geq 0$ for every $\omega$ with a strict inequality for some $\omega$, corresponding to the notion of one point arbitrage considered in Riedel (2015). In this setting, no arbitrage is equivalent to the existence a set of martingale measures $\mathcal{Q}_{\text {op }}$ so that for each point there exists $\mathbb{Q} \in \mathcal{Q}_{\text {op }}$ putting positive mass to that point.

Other authors (Burzoni, Frittelli, and Maggis (2016), Riedel (2015), Dolinsky and Soner (2014b)) introduced the notion of open arbitrage. In probability space frameworks, the family of null-sets defines "small," that is, negligible events. In absence of a reference probability, it might be still reasonable to distinguish large and small events. When there is a topology, one can define "small" events as those sets that are countable unions of closed sets with empty interior (Baire first category set). Open sets are then considered as relevant. We might then call $\ell \in \mathcal{I}$ an open arbitrage if it is nonnegative and is strictly positive on an open set. This case can be modeled in our framework by requiring the relevant claims to be continuous, nonnegative functions that are different from zero somewhere, that is,

$$
\mathcal{R}_{\text {open }}:=\left\{R \in C_{b}(\Omega) \cap \mathcal{P}: \exists \omega_{0} \in \Omega \text { such that } R\left(\omega_{0}\right)>0\right\} .
$$

It is then clear that when $R \in \mathcal{R}$ then it is nonzero on an open set.

Acciaio et al. (2016) defined a claim to be an arbitrage when it is positive everywhere, corresponding, in our model, to the choice

$$
\mathcal{R}_{+}:=\{R \in \mathcal{P}: R(\omega)>0, \forall \omega \in \Omega\} .
$$

\footnotetext{
${ }^{27}$ One might also compare the similar approach in Burzoni, Frittelli, and Maggis (2016).
} 
Bartl et al. (2017) considered a slightly stronger notion of relevant claims. Their choice is

$$
\mathcal{R}_{u}=\{R \in \mathcal{P}: \exists c \in(0, \infty) \text { such that } R \equiv c\} .
$$

Hence, $\ell \in \mathcal{I}$ is an arbitrage if is uniformly positive, which is sometimes called uniform arbitrage. Notice that with the choice $\mathcal{R}_{u}$, the notions of arbitrage and free lunch with vanishing risk are equivalent. ${ }^{28}$

\section{PROOF OF THE THEOREMS}

Let $(\mathcal{H}, \tau, \leq \mathcal{I}, \mathcal{R})$ be a given financial market. Recall that $(\mathcal{H}, \tau)$ is a metrizable topological vector space; we write $\mathcal{H}^{\prime}$ for its topological dual. We let $\mathcal{H}_{+}^{\prime}$ be the set of all positive functionals, that is, $\varphi \in \mathcal{H}_{+}^{\prime}$ provided that $\varphi(X) \geq 0$ for every $X \geq 0$ and $X \in \mathcal{H}$.

The following functional generalizes the notion of superreplication functional from the probabilistic to our order-theoretic framework. It plays a central role in our analysis. For $X \in \mathcal{H}$, let

$$
\begin{aligned}
\mathcal{D}(X):= & \inf \left\{c \in \mathbb{R}: \exists\left\{\ell_{n}\right\}_{n=1}^{\infty} \subset \mathcal{I},\left\{e_{n}\right\}_{n=1}^{\infty} \subset \mathcal{H}_{+}, e_{n} \stackrel{\tau}{\rightarrow} 0,\right. \\
& \text { such that } \left.c+e_{n}+\ell_{n} \geq X\right\} .
\end{aligned}
$$

Note that $\mathcal{D}$ is extended real valued. In particular, it takes the value $+\infty$ when there are no superreplicating portfolios. It might also take the value $-\infty$ if there is no lower bound.

We observe first that the absence of free lunches with vanishing risk can be equivalently described by the statement that the superreplication functional $\mathcal{D}$ assigns a strictly positive value to all relevant claims.

PROPOSITION 5.1: The financial market is strongly free of arbitrage if and only if $\mathcal{D}(R)>0$ for every $R \in \mathcal{R}$.

PROOF: Suppose $\left\{\ell_{n}\right\}_{n=1}^{\infty} \subset \mathcal{I}$ is a free lunch with vanishing risk. Then there is $R^{*} \in \mathcal{R}$ and $\left\{e_{n}\right\}_{n=1}^{\infty} \subset \mathcal{H}_{+}$with $e_{n} \stackrel{\tau}{\rightarrow} 0$ so that $e_{n}+\ell_{n} \geq R^{*}$. In view of the definition, we obtain $\mathcal{D}\left(R^{*}\right) \leq 0$.

To prove the converse, suppose that $\mathcal{D}\left(R^{*}\right) \leq 0$ for some $R^{*} \in \mathcal{R}$. Then the definition of $\mathcal{D}\left(R^{*}\right)$ implies that there is a sequence of real numbers $\left\{c_{k}\right\}_{k=1}^{\infty}$ with $c_{k} \downarrow \mathcal{D}\left(R^{*}\right)$, net trades $\left\{\ell_{k, n}\right\}_{n=1}^{\infty} \subset \mathcal{I}$, and $\left\{e_{k, n}\right\}_{n=1}^{\infty} \subset \mathcal{H}_{+}$with $e_{k, n} \stackrel{\tau}{\rightarrow} 0$ for $n \rightarrow \infty$ such that

$$
c_{k}+e_{k, n}+\ell_{k, n} \geq R^{*}, \quad \forall n, k \in \mathbb{N} .
$$

Let $B_{r}(0)$ be the ball with radius $r$ centered at zero with the metric compatible with $\tau$. For every $k$, choose $n=n(k)$ such that $e_{k, n} \in B_{\frac{1}{k}}(0)$. Set $\tilde{\ell}_{k}:=\ell_{k, n(k)}$ and $\tilde{e}_{k}:=e_{k, n(k)}+$

\footnotetext{
${ }^{28}$ The no arbitrage condition with $\mathcal{R}_{u}$ is the weakest one, $\mathcal{R}_{\mathrm{op}}$ is the strongest one. The first one is equivalent to the existence of one sublinear martingale expectation. The latter one is equivalent to the existence of a sublinear expectation that puts positive measure to all points. In general, the no-arbitrage condition based on $\mathcal{R}_{+}$is not equivalent to the absence of uniform arbitrage. However, absence of uniform arbitrage implies the existence of a linear bounded functional that is consistent with the market. In particular, risk neutral functionals are positive on $\mathcal{R}_{u}$. Moreover, if the set $\mathcal{I}$ is "large" enough then one can show that the risk neutral functionals give rise to countably additive measures. In Acciaio et al. (2016), this conclusion is achieved by using the so-called "power-option" placed in the set $\mathcal{I}$ as a static hedging possibility; compare also Bartl et al. (2017).
} 
$\left(c_{k} \vee 0\right)$. Then $\tilde{e}_{k}+\tilde{\ell}_{k} \geq R^{*}$ for every $k$. Since $\tilde{e}_{k} \stackrel{\tau}{\rightarrow} 0,\left\{\tilde{\ell}_{k}\right\}_{k=1}^{\infty}$ is a free lunch with vanishing risk.

It is clear that $\mathcal{D}$ is convex and we now use the tools of convex duality to characterize this functional in more detail. Recall the set of absolutely continuous martingale functionals $\mathcal{Q}_{\text {ac }}$ defined in Section 2 .

PROPOSITION 5.2: Assume that the financial market is strongly free of arbitrage. Then the superreplication functional $\mathcal{D}$ defined in (5.1) is a lower semi-continuous, sublinear martingale expectation with full support. Moreover,

$$
\mathcal{D}(X)=\sup _{\varphi \in \mathcal{Q}_{\mathrm{ac}}} \varphi(X), \quad X \in \mathcal{H} .
$$

The technical proof of this statement can be found in the Appendix. The important insight is that the superreplication functional can be described by a family of linear functionals. In the probabilistic setup, they correspond to the family of (absolutely continuous) martingale measures. With the help of this duality, we are now able to carry out the proof of our first main theorem.

Proof OF THEOREM 2.1: Suppose first that the market is viable and for some $R^{*} \in$ $\mathcal{R}$, there are sequences $\left\{e_{n}\right\}_{n=1}^{\infty} \subset \mathcal{H}_{+}$and $\left\{\ell_{n}\right\}_{n=1}^{\infty} \subset \mathcal{I}$ with $e_{n} \stackrel{\tau}{\rightarrow} 0$, and $e_{n}+\ell_{n} \geq R^{*}$. By viability, there is a family of agents $\left\{\preceq_{a}\right\}_{a \in A} \subset \mathcal{A}$ such that for some $a \in A$ we have $R^{*} \succ_{a} 0$. Since $\leq$ is a preorder compatible with the vector space operations, we have $-e_{n}+R^{*} \leq \ell_{n}$. As $\preceq_{a} \in \mathcal{A}$ is monotone with respect to $\leq$, we have $-e_{n}+R^{*} \preceq_{a} \ell_{n}$. By optimality of the zero trade, $\ell_{n} \preceq_{a} 0$, and we get $-e_{n}+R^{*} \preceq_{a} 0$. By lower semicontinuity of $\preceq_{a}$, we conclude that $R^{*} \preceq_{a} 0$, a contradiction.

Suppose now that the market is strongly free of arbitrage. By Proposition 5.1, $\mathcal{D}(R)>0$, for every $R \in \mathcal{R}$. In particular, this implies that the family $\mathcal{Q}_{\mathrm{ac}}$ is nonempty, as otherwise the supremum over $\mathcal{Q}_{\mathrm{ac}}$ would be $-\infty$. For each $\varphi \in \mathcal{Q}_{\mathrm{ac}}$, define $\preceq_{\varphi}$ by

$$
X \preceq_{\varphi} Y, \quad \Leftrightarrow \quad \varphi(X) \leq \varphi(Y) .
$$

One directly verifies that $\preceq_{\varphi} \in \mathcal{A}$. Moreover, $\varphi(\ell) \leq \varphi(0)=0$ for any $\ell \in \mathcal{I}$ implies that $\ell_{\varphi}^{*}=0$ is optimal for $\preceq_{\varphi}$ and (2.1) is satisfied. Finally, Proposition 5.1 and Proposition 5.2 imply that for any $R \in \mathcal{R}$, there exists $\varphi \in \mathcal{Q}_{\text {ac }}$ such that $\varphi(R)>0$; thus, (2.2) is satisfied. We deduce that $\left\{\preceq_{\varphi}\right\}_{\varphi \in \mathcal{Q}_{\mathrm{ac}}}$ supports the financial market $(\mathcal{H}, \tau, \leq, \mathcal{I}, \mathcal{R})$.

The previous arguments also imply our version of the fundamental theorem of asset pricing. In fact, with absence of arbitrage, the superreplication function is a lower semicontinuous sublinear martingale expectation with full support. Convex duality allows to prove the converse.

Proof of Theorem 2.2: Suppose the market is viable. From Theorem 2.1, it is strongly free of arbitrage. From Proposition 5.2, the superreplication functional is the desired lower semicontinuous sublinear martingale expectation with full support.

Suppose now that $\mathcal{E}$ is a lower semicontinuous sublinear martingale expectation with full support. In particular, $\mathcal{E}$ is a convex, lower semicontinuous, proper functional. Then, by the Fenchel-Moreau theorem,

$$
\mathcal{E}(X)=\sup _{\varphi \in \operatorname{dom}\left(\mathcal{E}^{*}\right)} \varphi(X),
$$


where $\operatorname{dom}\left(\mathcal{E}^{*}\right)=\left\{\varphi \in \mathcal{H}^{\prime}: \varphi(X) \leq \mathcal{E}(X), \forall X \in \mathcal{H}\right\}$. We can proceed as in the proof of Theorem 2.1 , to verify the viability of $(\mathcal{H}, \tau, \leq, \mathcal{I}, \mathcal{R})$ using the preference relations $\left\{\preceq_{\varphi}\right.$ \}$_{\varphi \in \operatorname{dom}\left(\mathcal{E}^{*}\right)}$.

We finally show the maximality of $\mathcal{E}_{\mathcal{Q}_{\mathrm{ac}}}$. Let $\mathcal{E}$ be a lower semicontinuous sublinear martingale expectation with full support. With the help of the martingale property of $\mathcal{E}$ one can show, as in Lemma A.5, that every $\varphi \in \operatorname{dom}\left(\mathcal{E}^{*}\right)$ is a martingale functional. As $\mathcal{E}$ is monotone with respect to $\leq$, we also conclude that $\varphi$ vanishes for negligible payoffs. Hence, we obtain $\operatorname{dom}\left(\mathcal{E}^{*}\right) \subset \mathcal{Q}_{\mathrm{ac}}$. From the above dual representation for $\mathcal{E}_{\mathcal{Q}_{\mathrm{ac}}}, \mathcal{E}(X) \leq$ $\mathcal{E}_{\mathcal{Q}_{\mathrm{ac}}}(X)$ for every $X \in \mathcal{H}$ follows.

Q.E.D.

\section{CONCLUSION}

This paper studies economic viability of a given financial market without assuming a probability-space framework. We show that it is possible to understand the equivalence of economic viability and the absence of arbitrage on the basis solely of a common order; the order (which is typically quite incomplete) is unanimous in the sense that agents' preferences are monotone with respect to it. A given financial market is viable if and only if a sublinear pricing functional exists that is consistent with the given asset prices.

The properties of the common order are reflected in expected equilibrium returns. When the common order is given by the expected value under some common prior, expected returns under that prior have to be equal in equilibrium, and thus, Fama's efficient market hypothesis results. If the common order is determined by the almost sure order under some common prior, we obtain the weak form of the efficient market hypothesis that states that expected returns are equal under some (martingale) measure that shares the same null sets as the common prior.

In situations of Knightian uncertainty, it might be too demanding to impose a common prior for all agents. When Knightian uncertainty is described by a class of priors, it is necessary to replace the linear (martingale) expectation by a sublinear expectation. It is then no longer possible to reach the conclusion that expected returns are equal under some probability measure. Knightian uncertainty might thus be an explanation for empirical violations of the efficient market hypothesis. In particular, there is always a range of economically justifiable arbitrage-free prices. In this sense, Knightian uncertainty shares similarities with markets with friction or that are incomplete, but the economic reason for the price indeterminacy is different.

\section{APPENDIX: PROOF OF PROPOSITION 5.2}

We separate the proof in several steps. Recall that the super-replication functional $\mathcal{D}$ is defined in (5.1).

LEMma A.1: Assume that the financial market is strongly free of arbitrage. Then $\mathcal{D}$ is convex, lower semicontinuous and $\mathcal{D}(X)>-\infty$ for every $X \in \mathcal{H}$.

Proof: The convexity of $\mathcal{D}$ follows immediately from the definitions. To prove lower semi-continuity, consider a sequence $X_{k} \stackrel{\tau}{\rightarrow} X$ with $\mathcal{D}\left(X_{k}\right) \leq c$. Then, by definition, for every $k$ there exists a sequence $\left\{e_{k, n}\right\}_{n=1}^{\infty} \subset \mathcal{H}_{+}$with $e_{k, n} \stackrel{\tau}{\rightarrow} 0$ for $n \rightarrow \infty$ and a sequence $\left\{\ell_{k, n}\right\}_{n=1}^{\infty} \subset \mathcal{I}$ such that $c+\frac{1}{k}+e_{k, n}+\ell_{k, n} \geq X_{k}$, for every $k, n$. Let $B_{r}(0)$ be the ball of radius $r$ centered around zero in the metric compatible with $\tau$. Choose $n=n(k)$ such that $e_{k, n} \in B_{\frac{1}{k}}$ and set $\tilde{e}_{k}:=e_{k, n(k)}, \tilde{\ell}_{k}:=\ell_{k, n(k)}$. Then $c+\frac{1}{k}+\tilde{e}_{k}+\left(X-X_{k}\right)+\tilde{\ell}_{k} \geq X$ 
and $\frac{1}{k}+\tilde{e}_{k}+\left(X-X_{k}\right) \stackrel{\tau}{\rightarrow} 0$ as $k \rightarrow \infty$. Hence, $\mathcal{D}(X) \leq c$. This proves that $\mathcal{D}$ is lower semicontinuous.

The constant claim 1 is relevant and by Proposition 5.1, $\mathcal{D}(1) \in(0,1]$; in particular, it is finite. Toward a counterposition, suppose that there exists $X \in \mathcal{H}$ such that $\mathcal{D}(X)=-\infty$. For $\lambda \in[0,1]$, set $X_{\lambda}:=X+\lambda(1-X)$. The convexity of $\mathcal{D}$ implies that $\mathcal{D}\left(X_{\lambda}\right)=-\infty$ for every $\lambda \in[0,1)$. Since $\mathcal{D}$ is lower semicontinuous, $0<\mathcal{D}(1) \leq \lim _{\lambda \rightarrow 1} \mathcal{D}\left(X_{\lambda}\right)=-\infty$, a contradiction.

LEMma A.2: Assume that the financial market is strongly free of arbitrage. The superreplication functional $\mathcal{D}$ is a sublinear expectation with full-support. Moreover, $\mathcal{D}(c)=c$ for every $c \in \mathbb{R}$, and

$$
\mathcal{D}(X+\ell) \leq \mathcal{D}(X), \quad \forall \ell \in \mathcal{I}, X \in \mathcal{H} .
$$

In particular, $\mathcal{D}$ has the martingale property.

PROOF: We prove this result in two steps.

Step 1. In this step, we prove that $\mathcal{D}$ is a sublinear expectation. Let $X, Y \in \mathcal{H}$ such that $X \leq Y$. Suppose that there are $c \in \mathbb{R},\left\{\ell_{n}\right\}_{n=1}^{\infty} \subset \mathcal{I}$ and $\left\{e_{n}\right\}_{n=1}^{\infty} \subset \mathcal{H}_{+}$with $e_{n} \stackrel{\tau}{\rightarrow} 0$ satisfying, $Y \leq c+e_{n}+\ell_{n}$. Then, from the transitivity of $\leq$, we also have $X \leq c+e_{n}+\ell_{n}$. Hence, $\mathcal{D}(X) \leq \mathcal{D}(Y)$, and consequently $\mathcal{D}$ is monotone with respect to $\leq$.

Translation-invariance, $\mathcal{D}(c+g)=c+\mathcal{D}(g)$, follows directly from the definitions.

We next show that $\mathcal{D}$ is sub-additive. Fix $X, Y \in \mathcal{H}$. Suppose that either $\mathcal{D}(X)=\infty$ or $\mathcal{D}(Y)=\infty$. Then, since by Lemma A.1 $\mathcal{D}>-\infty$, we have $\mathcal{D}(X)+\mathcal{D}(Y)=\infty$ and the sub-additivity follows directly. Now we consider the case $\mathcal{D}(X), \mathcal{D}(Y)<\infty$. Hence, there are $c_{X}, c_{Y} \in \mathbb{R},\left\{\ell_{n}^{X}\right\}_{n=1}^{\infty},\left\{\ell_{n}^{Y}\right\}_{n=1}^{\infty} \subset \mathcal{I}$ and $\left\{e_{n}^{X}\right\}_{n=1}^{\infty},\left\{e_{n}^{Y}\right\}_{n=1}^{\infty} \subset \mathcal{H}_{+}$with $e_{n}^{X}, e_{n}^{Y} \stackrel{\sim}{\rightarrow} 0$ satisfying,

$$
c_{X}+\ell_{n}^{X}+e_{n}^{X} \geq X, \quad c_{Y}+\ell_{n}^{Y}+e_{n}^{Y} \geq Y .
$$

Set $\bar{c}:=c_{X}+c_{Y}, \bar{\ell}_{n}:=\ell_{n}^{X}+\ell_{n}^{Y}, \bar{e}_{n}:=e_{n}^{X}+e_{n}^{Y}$. Since $\mathcal{I}$ is a positive cone, $\left\{\bar{\ell}_{n}\right\}_{n=1}^{\infty} \subset \mathcal{I}$, $\bar{e}_{n} \stackrel{\tau}{\rightarrow} 0$ and

$$
\bar{c}+\bar{e}_{n}+\bar{\ell}_{n} \geq X+Y \quad \Rightarrow \quad \mathcal{D}(X+Y) \leq \bar{c} .
$$

Since this holds for any such $c_{X}, c_{Y}$, we conclude that

$$
\mathcal{D}(X+Y) \leq \mathcal{D}(X)+\mathcal{D}(Y) .
$$

Finally, we show that $\mathcal{D}$ is positively homogeneous of degree one. Suppose that $c+e_{n}+$ $\ell_{n} \geq X$ for some constant $c,\left\{\ell_{n}\right\}_{n=1}^{\infty} \subset \mathcal{I}$ and $\left\{e_{n}\right\}_{n=1}^{\infty} \subset \mathcal{H}_{+}$with $e_{n} \stackrel{\tau}{\rightarrow} 0$. Then, for any $\lambda>0$ and for any $n \in \mathbb{N}, \lambda c+\lambda e_{n}+\lambda \ell_{n} \geq \lambda X$. Since $\lambda \ell_{n} \in \mathcal{I}$ and $\lambda e_{n} \stackrel{\tau}{\rightarrow} 0$, this implies that

$$
\mathcal{D}(\lambda X) \leq \lambda \mathcal{D}(X), \quad \lambda>0, X \in \mathcal{H} .
$$

Notice that above holds trivially when $\mathcal{D}(X)=+\infty$. Conversely, if $\mathcal{D}(\lambda X)=+\infty$ we are done. Otherwise, we use (A.2) with $\lambda X$ and $1 / \lambda$,

$$
\mathcal{D}(X)=\mathcal{D}\left(\frac{1}{\lambda} \lambda X\right) \leq \frac{1}{\lambda} \mathcal{D}(\lambda X), \quad \Rightarrow \quad \lambda \mathcal{D}(X) \leq \mathcal{D}(\lambda X) .
$$

Hence, $\mathcal{D}$ positively homogeneous and it is a sublinear expectation. 
Step 2. In this step, we assume that the financial market is strongly free of arbitrages. Since $0 \in \mathcal{I}$, we have $\mathcal{D}(0) \leq 0$. If the inequality is strict, we obviously have a free lunch with vanishing risk,; hence $\mathcal{D}(0)=0$ and from translation-invariance the same applies to every $c \in \mathbb{R}$. Moreover, by Proposition 5.1, $\mathcal{D}$ has full support. Thus, we only need to prove (A.1).

Suppose that $X \in \mathcal{H}, \ell \in \mathcal{I}$ and $c+e_{n}+\ell_{n}^{X} \geq X$. Since $\mathcal{I}$ is a convex cone, $\ell_{n}^{X}+\ell \in \mathcal{I}$ and $c+e_{n}+\left(\ell+\ell_{n}^{X}\right) \geq X+\ell$. Therefore, $\mathcal{D}(X+\ell) \leq c$. Since this holds for all such constants, we conclude that $\mathcal{D}(X+\ell) \leq \mathcal{D}(X)$ for all $X \in \mathcal{H}$. In particular, $\mathcal{D}(\ell) \leq 0$ and the martingale property is satisfied.

Q.E.D.

REMARK A.3: Note that for $\mathcal{H}=\left(\mathcal{B}_{b},\|\cdot\|_{\infty}\right)$, the definition of $\mathcal{D}$ reduces to the classical one:

$$
\mathcal{D}(X):=\inf \{c \in \mathbb{R}: \exists \ell \in \mathcal{I} \text {, such that } c+\ell \geq X\} .
$$

Indeed, if $c+\ell \geq X$ for some $c$ and $\ell$, one can use the constant sequences $\ell_{n} \equiv \ell$ and $e_{n} \equiv 0$ to get that $\mathcal{D}$ in (5.1) is less or equal than the one in (A.3). For the converse inequality, observe that if $c+e_{n}+\ell_{n} \geq X$ for some $c, \ell_{n}$, and $e_{n}$ with $\left\|e_{n}\right\|_{\infty} \rightarrow 0$, then the infimum in (A.3) is less or equal than $c$. The thesis follows. Lemma A.1 is in line with the well-known fact that the classical superreplication functional in $\mathcal{B}_{b}$ is Lipschitz continuous with respect to the sup-norm topology.

The results of Lemma A.1 and Lemma A.2 imply that the super-replication functional defined in (5.1) is a proper convex function in the language of convex analysis, compare, for example, Rockafellar (2015). By the classical Fenchel-Moreau theorem, we have the following dual representation of $\mathcal{D}$ :

$$
\begin{array}{ll}
\mathcal{D}(X)=\sup _{\varphi \in \mathcal{H}^{\prime}}\left\{\varphi(X)-\mathcal{D}^{*}(\varphi)\right\}, \quad X \in \mathcal{H}, \quad \text { where } \\
\mathcal{D}^{*}(\varphi)=\sup _{Y \in \mathcal{H}}\{\varphi(Y)-\mathcal{D}(Y)\}, \quad \varphi \in \mathcal{H}^{\prime} .
\end{array}
$$

Since $\varphi(0)=\mathcal{D}(0)=0, \mathcal{D}^{*}(\varphi) \geq \varphi(0)-\mathcal{D}(0)=0$ for every $\varphi \in \mathcal{H}^{\prime}$. However, it may take the value plus infinity. Set,

$$
\operatorname{dom}\left(\mathcal{D}^{*}\right):=\left\{\varphi \in \mathcal{H}^{\prime}: \mathcal{D}^{*}(\varphi)<\infty\right\} .
$$

LEMMA A.4: We have

$$
\operatorname{dom}\left(\mathcal{D}^{*}\right)=\left\{\varphi \in \mathcal{H}_{+}^{\prime}: \mathcal{D}^{*}(\varphi)=0\right\}=\left\{\varphi \in \mathcal{H}_{+}^{\prime}: \varphi(X) \leq \mathcal{D}(X), \forall X \in \mathcal{H}\right\} .
$$

In particular,

$$
\mathcal{D}(X)=\sup _{\varphi \in \operatorname{dom}\left(\mathcal{D}^{*}\right)} \varphi(X), \quad X \in \mathcal{H} .
$$

Furthermore, there are free lunches with vanishing risk in the financial market, whenever $\operatorname{dom}\left(\mathcal{D}^{*}\right)$ is empty.

PROOF: Clearly, the two sets on the right of (A.4) are equal and included in $\operatorname{dom}\left(\mathcal{D}^{*}\right)$. The definition of $\mathcal{D}^{*}$ implies that

$$
\varphi(X) \leq \mathcal{D}(X)+\mathcal{D}^{*}(\varphi), \quad \forall X \in \mathcal{H}, \varphi \in \mathcal{H}^{\prime} .
$$


By homogeneity,

$$
\varphi(\lambda X) \leq \mathcal{D}(\lambda X)+\mathcal{D}^{*}(\varphi), \quad \Rightarrow \quad \varphi(X) \leq \mathcal{D}(X)+\frac{1}{\lambda} \mathcal{D}^{*}(\varphi),
$$

for every $\lambda>0$ and $X \in \mathcal{H}$. Suppose that $\varphi \in \operatorname{dom}\left(\mathcal{D}^{*}\right)$. We then let $\lambda$ go to infinity to arrive at $\varphi(X) \leq \mathcal{D}(X)$ for all $X \in \mathcal{B}_{b}$. Hence, $\mathcal{D}^{*}(\varphi)=0$.

Fix $X \in \mathcal{H}_{+}$. Since $\leq$is monotone with respect to the pointwise order, $-X \leq 0$. Then, by the monotonicity of $\mathcal{D}, \varphi(-X) \leq \mathcal{D}(-X) \leq \mathcal{D}(0) \leq 0$. Hence, $\varphi \in \mathcal{H}_{+}^{\prime}$.

Now suppose that $\operatorname{dom}\left(\mathcal{D}^{*}\right)$ is empty or, equivalently, $\mathcal{D}^{*} \equiv \infty$. Then the dual representation implies that $\mathcal{D} \equiv-\infty$. In view of Proposition 5.1, there are free lunches with vanishing risk in the financial market.

Q.E.D.

We next show that, under the assumption of absence of free lunch with vanishing risk with respect to any $\mathcal{R}$, the set $\operatorname{dom}\left(\mathcal{D}^{*}\right)$ is equal to $\mathcal{Q}_{\text {ac }}$ defined in Section 2 . Since any relevant set $\mathcal{R}$ by hypothesis contains $\mathcal{R}_{u}$ defined in (4.1), to obtain this conclusion it would be sufficient to assume the absence of free lunch with vanishing risk with respect to any $\mathcal{R}_{u}$.

LEMMA A.5: Suppose the financial market is strongly free of arbitrage with respect to $\mathcal{R}$. Then $\operatorname{dom}\left(\mathcal{D}^{*}\right)$ is equal to the set of absolutely continuous martingale functionals $\mathcal{Q}_{\mathrm{ac}}$.

ProOF: The fact that $\operatorname{dom}\left(\mathcal{D}^{*}\right)$ is nonempty follows from Lemma A.2 and Lemma A.4. Fix an arbitrary $\varphi \in \operatorname{dom}\left(\mathcal{D}^{*}\right)$. By Lemma A.2, $\mathcal{D}(c)=c$ for every constant $c \in \mathbb{R}$. In view of the dual representation of Lemma A.4,

$$
c \varphi(1)=\varphi(c) \leq \mathcal{D}(c)=c, \quad \forall c \in \mathbb{R} .
$$

Hence, $\varphi(1)=1$.

We continue by proving the monotonicity property. Suppose that $X \in \mathcal{P}$. Since $0 \in \mathcal{I}$, we obviously have $\mathcal{D}(-X) \leq 0$. The dual representation implies that $\varphi(-X) \leq$ $\mathcal{D}(-X) \leq 0$. Thus, $\varphi(X) \geq 0$.

We now prove the supermartingale property. Let $\ell \in \mathcal{I}$. Obviously, $\mathcal{D}(\ell) \leq 0$. By the dual representation, $\varphi(\ell) \leq \mathcal{D}(\ell) \leq 0$. Hence $\varphi$ is a martingale functional. The absolute continuity follows as in Lemma S.4.3. Hence, $\varphi \in \mathcal{Q}_{\text {ac }}$.

To prove the converse, fix an arbitrary $\varphi \in \mathcal{Q}_{\text {ac }}$. Suppose that $X \in \mathcal{H}, c \in \mathbb{R},\left\{\ell_{n}\right\}_{n=1}^{\infty} \subset \mathcal{I}$ and $\left\{e_{n}\right\}_{n=1}^{\infty} \subset \mathcal{H}_{+}$with $e_{n} \stackrel{\tau}{\rightarrow} 0$ satisfy, $c+e_{n}+\ell_{n} \geq X$. From the properties of $\varphi$,

$$
0 \leq \varphi\left(c+e_{n}+\ell_{n}-X\right)=\varphi\left(c+e_{n}-X\right)+\varphi\left(\ell_{n}\right) \leq c-\varphi\left(X-e_{n}\right) .
$$

Since $e_{n} \stackrel{\tau}{\rightarrow} 0$ and $\varphi$ is continuous, $\varphi(X) \leq \mathcal{D}(X)$ for every $X \in \mathcal{H}$. Therefore, $\varphi \in$ $\operatorname{dom}\left(\mathcal{D}^{*}\right)$.

Q.E.D.

Proof OF Proposition 5.2: It follows directly from Lemma A.4 and Lemma A.5. Q.E.D.

We have the following immediate corollary, which proves the first part of the fundamental theorem of asset pricing in this context.

COROLlARY A.6: The financial market is strongly free of arbitrage if and only $\mathcal{Q}_{\mathrm{ac}} \neq \emptyset$ and for any $R \in \mathcal{R}$, there exists $\varphi_{R} \in \mathcal{Q}_{\mathrm{ac}}$ such that $\varphi_{R}(R)>0$. 
PROOF: By contradiction, suppose that there exists $R^{*}$ such that $e_{n}+\ell_{n} \geq R^{*}$ with $e_{n} \stackrel{\tau}{\rightarrow} 0$. Take $\varphi_{R^{*}}$ such that $\varphi_{R^{*}}\left(R^{*}\right)>0$ and observe that $0<\varphi_{R^{*}}\left(R^{*}\right) \leq \varphi\left(e_{n}+\ell_{n}\right) \leq$ $\varphi\left(e_{n}\right)$. Since $\varphi \in \mathcal{H}_{+}^{\prime}, \varphi\left(e_{n}\right) \rightarrow 0$ as $n \rightarrow \infty$, which is a contradiction.

In the other direction, assume that the financial market is strongly free of arbitrage. By Lemma A.5, $\operatorname{dom}\left(\mathcal{D}^{*}\right)=\mathcal{Q}_{\text {ac }}$. Let $R \in \mathcal{R}$ and note that, by Proposition 5.1, $\mathcal{D}(R)>0$. It follows that there exists $\varphi_{R} \in \operatorname{dom}\left(\mathcal{D}^{*}\right)=\mathcal{Q}_{\text {ac }}$ satisfying $\varphi_{R}(R)>0$.

Q.E.D.

REMARK A.7: The set of positive functionals $\mathcal{Q}_{\mathrm{ac}} \subset \mathcal{H}_{+}^{\prime}$ is the analogue of the set of local martingale measures of the classical setting. Indeed, all elements of $\varphi \in \mathcal{Q}_{\mathrm{ac}}$ can be regarded as supermartingale "measures," since $\varphi(\ell) \leq 0$ for every $\ell \in \mathcal{I}$. Moreover, the property $\varphi(Z)=0$ for every $Z \in \mathcal{Z}$ can be regarded as absolute continuity with respect to null sets. The full support property is our analog to the converse absolute continuity. However, the full-support property cannot be achieved by a single element of $\mathcal{Q}_{\text {ac }}$.

Bouchard and Nutz (2015) studied arbitrage for a set of priors $\mathcal{M}$. The absolute continuity and the full support properties then translate to the statement that " $\mathcal{M}$ and $\mathcal{Q}$ have the same polar sets." In the paper by Burzoni, Frittelli, and Maggis (2016), a class of relevant sets $\mathcal{S}$ is given and the two properties can summarized by the statement "the set $\mathcal{S}$ is not contained in the polar sets of $\mathcal{Q}$."

Also, when $\mathcal{H}=\mathcal{B}_{b}, \mathcal{H}^{\prime}$ is the class of bounded additive measures $b a$. It is a classical question whether one can restrict $\mathcal{Q}$ to the set of countable additive measures $c a_{r}(\Omega)$. In several of the examples described in Section 4 and 3, this is proved. However, there are examples for which this is not true.

\section{REFERENCES}

Acciaio, B., M. Beiglböck, F. Penkner, And W. Schachermayer (2016): "A Model-Free Version of the Fundamental Theorem of Asset Pricing and the Super-Replication Theorem," Mathematical Finance, 26, 233-251. [1209,1225,1226]

Aliprantis, C. D., AND K. C. Border (1999): Infinite-Dimensional Analysis. Springer. [1221]

Araujo, A., A. Chateauneuf, And J. H. Faro (2018): "Financial Market Structures Revealed by Pricing Rules: Efficient Complete Markets Are Prevalent,” Journal of Economic Theory, 173, 257-288. [1223]

ARROw, K. (1953): "[”Fr]Le rôle des valeurs boursières pour la répartition la meilleure des risques, in Économétrie, Colloques Internationaux du Centre National de la Recherche Scientifique. Centre National de la Recherche Scientifique. [1210]

ARtzner, P., F. Delbaen, J.-M. Eber, AND D. HeAth (1999): “Coherent Measures of Risk,” Mathematical Finance, 9, 203-228. [1208,1213]

BACHELIER, L. (1900): "Théorie de la Spéculation," Annales Scientifiques de l'École Normale Supérieure, 21-86. [1216]

BARTL, D. (2019): "Exponential Utility Maximization Under Model Uncertainty for Unbounded Endowments," The Annals of Applied Probability, 29, 577-612. [1214]

BARTL, D., P. Cheridito, M. KuPPER, AND L. TANGPI (2017): "Duality for Increasing Convex Functionals With Countably Many Marginal Constraints," Banach Journal of Mathematical Analysis, 11, 72-89. [1209, 1226]

BEISSNER, P., AND L. DENIS (2018): "Duality and General Equilibrium Theory Under Knightian Uncertainty," SIAM Journal on Financial Mathematics, 9, 381-400. [1219]

BEISSNER, P., AND F. Riedel (2019): “Equilibria Under Knightian Price Uncertainty,” Econometrica, 87, $37-$ 64. $[1208,1220,1224]$

Bewley, T. (2002): "Knightian Decision Theory: Part I," Decisions in Economics and Finance, 25, 79-110. [1218]

BiOn-NAdAl, J., M. KeRvarec et al. (2012): "Risk Measuring Under Model Uncertainty," The Annals of Applied Probability, 22, 213-238. [1219]

BoUCHARD, B., AND M. NuTZ (2015): "Arbitrage and Duality in Nondominated Discrete-Time Models," The Annals of Applied Probability, 25, 823-859. [1232] 
Burzoni, M., M. Frittelli, Z. Hou, M. MAgGis, AND J. ObŁÓJ (2019): “Pointwise Arbitrage Pricing Theory in Discrete Time," Mathematics of Operations Research, 44, 1034-1057. [1209]

Burzoni, M., M. FritTeldi, AND M. MAGgIS (2016): "Universal Arbitrage Aggregator in Discrete-Time Markets Under Uncertainty," Finance and Stochastics, 20, 1-50. [1225,1232]

Burzoni, M., F. RiEdel H. M. SONER (2021): "Supplement to 'Viability and Arbitrage Under Knightian Uncertainty'," Econometrica Supplemental Material, 89, https://doi.org/10.3982/ECTA16535. [1210]

CASSESE, G. (2017): "Asset Pricing in an Imperfect World," Economic Theory, 64, 539-570. [1209]

DALANG, R. C., A. MorTON, AND W. Willinger (1990): "Equivalent Martingale Measures and No-Arbitrage in Stochastic Securities Market Models," Stochastics: An International Journal of Probability and Stochastic Processes, 29, 185-201. [1209,1218]

DANA, R.-A., AND F. RIEDEL (2013): “Intertemporal Equilibria With Knightian Uncertainty,” Journal of Economic Theory, 148, 1582-1605. [1220]

DANA, R.-A., C. LE VAn, AND F. MAGNien (1999): “On the Different Notions of Arbitrage and Existence of Equilibrium,” Journal of Economic Theory, 87, 169-193. [1209]

DELBAEN, F., AND W. SCHACHERMAYER (1998): "The Fundamental Theorem of Asset Pricing for Unbounded Stochastic Processes," Mathematische Annalen, 312, 215-250. [1209,1211]

DOLINSKY, Y., AND H. M. SONER (2014a): "Martingale Optimal Transport and Robust Hedging in Continuous Time," Probability Theory and Related Fields, 160, 391-427. [1223]

\section{7. [1225]}

_ (2015): "Martingale Optimal Transport in the Skorokhod Space," Stochastic Processes and Their Applications, 125, 3893-3931. [1223]

DUFFIE, D., AND C.-F. HuANG (1985): “Implementing Arrow-Debreu Equilibria by Continuous Trading of few Long-Lived Securities," Econometrica, 1337-1356. [1209]

Eichberger, J., AND D. Kelsey (1999): "E-Capacities and the Ellsberg Paradox,” Theory and Decision, 46, 107-138. [1216]

EPSTEIN, L., AND S. Ji (2013): “Ambiguous Volatility and Asset Pricing in Continuous Time," Review of Financial Studies, 26, 1740-1786. [1208,1215,1222]

FAMA, E. F. (1970): "Efficient Capital Markets: A Review of Theory and Empirical Work," The Journal of Finance, 25, 383-417. [1208,1216]

FöllmER, H., AND A. SCHIED (2011): Stochastic Finance: An Introduction in Discrete Time. Walter de Gruyter. $[1208,1218]$

Ghysels, E., A. C. Harvey, And E. Renault (1996): "Stochastic Volatility," in Handbook of Statistics, Vol. 14, ed. by G. Maddala and C. Rao. Elsevier, 119-191. [1215]

GILBOA, I., AND D. SCHMEIDLER (1989): "Maxmin Expected Utility With Non-Unique Prior," Journal of Mathematical Economics, 18, 141-153. [1208,1218,1224]

GilboA, I., F. MACCHERONI, M. MARINACCI, AND D. SCHMEIDLER (2010): "Objective and Subjective Rationality in a Multiple Prior Model," Econometrica, 78, 755-770. [1218]

HAlmos, P. R., AND L. J. SAVAGE (1949): "Application of the Radon-Nikodym Theorem to the Theory of Sufficient Statistics," The Annals of Mathematical Statistics, 20, 225-241. [1218]

Hansen, L., AND T. J. SARgent (2001): "Robust Control and Model Uncertainty," American Economic Review, 91, 60-66. [1209]

HANSEN, L. P., AND T. J. SARgent (2008): Robustness. Princeton University Press. [1209]

HARRISON, J. M., AND D. M. KREPS (1979): "Martingales and Arbitrage in Multiperiod Securities Markets," Journal of Economic Theory, 20, 381-408. [1207,1209-1211,1218,1223,1224]

HARRISON, J. M., AND S. R. PLISKA (1981): "Martingales and Stochastic Integrals in the Theory of Continuous Trading," Stochastic Processes and Their Applications, 11, 215-260. [1209]

HESTON, S. L. (1993): "A Closed-Form Solution for Options With Stochastic Volatility With Applications to Bond and Currency Options," The Review of Financial Studies, 6, 327-343. [1215]

Huber, P. J. (1965): "A Robust Version of the Probability Ratio Test," The Annals of Mathematical Statistics, 1753-1758. [1216]

- (1981): Robust Statistics. Wiley Series in Probability and Mathematical Statistics. Wiley. [1208]

JARROW, R. A., AND M. LARSSON (2012): "The Meaning of Market Efficiency," Mathematical Finance: An International Journal of Mathematics, Statistics and Financial Economics, 22, 1-30. [1217]

JOUINI, E., AND H. KALLAL (1995): "Martingales and Arbitrage in Securities Markets With Transaction Costs," Journal of Economic Theory, 66, 178-197. [1209,1223]

(1999): "Viability and Equilibrium in Securities Markets With Frictions," Mathematical Finance, 9, 275-292. [1209] 
Klibanoff, P., M. MARinACCI, AND S. MukerJi (2005): "A Smooth Model of Decision Making Under Ambiguity," Econometrica, 73, 1849-1892. [1209,1218,1221]

KNIGHT, F. H. (1921): Risk, Uncertainty, and Profit. Library of Economics and Liberty. [1207]

KREPS, D. M. (1981): "Arbitrage and Equilibrium in Economies With Infinitely Many Commodities," Journal of Mathematical Economics, 8, 15-35. [1211,1224]

LeRoy, S. F., AND J. Werner (2014): Principles of Financial Economics. Cambridge University Press. [1223]

LO, A. W., AND M. Mueller (2010): “WARNING: Physics Envy May Be Hazardous to Your Wealth,”Journal of Investment Management, 8, 13-63. [1208]

LutTMER, E. (1996): “Asset Pricing in Economies With Frictions,” Econometrica, 64, 1439-1467. [1223]

Maccheroni, F., M. Marinacci, And A. Rustichini (2006): "Ambiguity Aversion, Robustness, and the Variational Representation of Preferences," Econometrica, 74, 1447-1498. [1208]

MALKIEL, B. G. (2003): "The Efficient Market Hypothesis and Its Critics," Journal of Economic Perspectives, 17, 59-82. [1216]

RIEDEL, F. (2015): "Financial Economics Without Probabilistic Prior Assumptions," Decisions in Economics and Finance, 38, 75-91. [1209,1225]

RigotTi, L., AND C. SHANNON (2005): "Uncertainty and Risk in Financial Markets," Econometrica, 73, 203243. [1220]

RockAfellar, R. T. (2015): Convex Analysis. Princeton University Press. [1230]

SAmuelson, P. (1965): "Proof That Properly Anticipated Prices Fluctuate Randomly," Industrial Management Review, 41-49. [1216]

SAmuelson, P. A. (1973): "Proof That Properly Discounted Present Values of Assets Vibrate Randomly," The Bell Journal of Economics and Management Science, 369-374. [1216]

SCHMEIDLER, D. (1989): "Subjective Probability and Expected Utility Without Additivity," Econometrica, 571587. [1224]

Soner, H. M., N. TOUZI, AND J. ZHANG (2012): "Wellposedness of Second Order Backward SDEs," Probability Theory and Related Fields, 153, 149-190. [1215]

(2013): "Dual Formulation of Second Order Target Problems," The Annals of Applied Probability, 23, 308-347. [1215]

VORBRINK, J. (2014): “Financial Markets With Volatility Uncertainty," Journal of Mathematical Economics, 53, 64-78. [1215]

WERnER, J. (1987): “Arbitrage and the Existence of Competitive Equilibrium," Econometrica, 1403-1418. [1209]

\section{Co-editor Bart Lipman handled this manuscript.}

Manuscript received 23 July, 2018; final version accepted 23 November, 2020; available online 3 December, 2020. 